\title{
Results on Optimal Biorthogonal Filter Banks
}

\author{
P. P. Vaidyanathan, Fellow, IEEE, and Ahmet Kirac, Student Member, IEEE
}

(Invited Paper)

\begin{abstract}
Optimization of filter banks for specific input statistics has been of interest in the theory and practice of subband coding. For the case of orthonormal filter banks with infinite order and uniform decimation, the problem has been completely solved in recent years. For the case of biorthogonal filter banks, significant progress has been made recently, although a number of issues still remain to be addressed. In this paper we briefly review the orthonormal case, and then present several new results for the biorthogonal case. All discussions pertain to the infinite order (ideal filter) case. The current status of research as well as some of the unsolved problems are described.
\end{abstract}

\section{INTRODUCTION}

$\mathbf{T}$ HE $M$-channel filter bank or subband coder (SBC) shown in Fig. 1(a) is commonly used in data compression for lossy encoding of audio and image signals. The decimators $\downarrow M$ and expanders $\uparrow M$ have the standard meanings which can be found in [13], [23], and [26]. The boxes labeled $Q$ represent uniform scalar quantizers. Fig. 1(b) shows the associated polyphase representation [23]. The additive noise sources $q_{i}(n)$ indicate the effect of subband quantizers. The system is said to be a biorthogonal filter bank if $\mathbf{R}\left(e^{j \omega}\right) \mathbf{E}\left(e^{j \omega}\right)=\mathbf{I}$ for all $\omega$. This implies perfect reconstruction, i.e., $\hat{x}(n)=x(n)$, in the absence of quantizers. The subband coder is orthonormal or paraunitary if $\mathbf{E}\left(e^{j \omega}\right)$ is unitary for all $\omega$. In this case biorthogonality is achieved by setting $\mathbf{R}\left(e^{j \omega}\right)=\mathbf{E}^{\dagger}\left(e^{j \omega}\right)$ or, equivalently, $F_{i}\left(e^{j \omega}\right)=H_{i}^{*}\left(e^{j \omega}\right)$.

When the subband quantizers are present in a biorthogonal filter bank, and if the average bit rate $b$ is fixed, what is the best choice of the filters $\left\{H_{i}\left(e^{j \omega}\right)\right\}$ [equivalently, the polyphase matrix $\left.\mathbf{E}\left(e^{j \omega}\right)\right]$, which minimizes the average (mean-squared) value of the reconstruction error $\hat{x}(n)-x(n)$ ? This is the theoretically optimal biorthogonal SBC problem. The special case, where $\mathbf{E}\left(e^{j \omega}\right)$ is memoryless (i.e., $\mathbf{E}\left(e^{j \omega}\right)$ is a constant matrix $\mathbf{T}$ ), is the transform coder problem, and was addressed by Huang and Schultheiss in 1963 [10], and in greater detail by Segall in 1976 [18]. The main outcomes of those papers are the optimum bit allocation formulas for the subband quantizers and the derivation of the KLT matrix as the optimum solution for $\mathbf{T}$. The KLT is a unitary matrix such that any pair of decimated subband signals $y_{i}(n), y_{m}(n)$ are uncorrelated for each $n$. The statistical assumptions used in the derivation were that the input is a Gaussian process and

Manuscript received August 30, 1997; revised November 17, 1997. This work was supported in part by the National Science Foundation under Grant MIP 0703755 and by Tektronix, Inc.

The authors are with the Department of Electrical Engineering, California Institute of Technology, Pasadena, CA 91125 USA.

Publisher Item Identifier S 1057-7130(98)04696-5. that the subband quantizers are optimal (nonuniform) scalar quantizers operating independently of each other. ${ }^{1}$

For the case of subband coders, $\mathbf{E}\left(e^{j \omega}\right)$ has memory, i.e., $\mathbf{E}(z)=\sum_{n} \mathbf{E}_{n} z^{-n}$, because the filters $\left\{H_{i}\left(e^{j \omega}\right)\right\}$ can have arbitrarily large orders. For this case, there exist several practical methods which numerically optimize the filter coefficients for minimizing the reconstruction error [3], [5], [12], [14], [20] (more generally a linear combination of a number of practical criteria, e.g., see [3]). Theoretical results on optimization of two channel orthonormal filter banks were developed by Unser in [21], and the closely related idea of principal component filter banks introduced and developed in [19] and [28]. A general set of necessary and sufficient conditions for the optimality of an orthonormal filter bank were independently developed in [24] and [25] for the case of unconstrained filter orders. For nonuniform filter banks (e.g., tree sturctured) the asymptotic performance has been analyzed in [6] and an entropy analysis reported in [16].

The fact that biorthogonal SBC has an advantage over orthonormal SBC for practical coding has been amply demonstrated in a number of papers, e.g., [4] and [2]. The theoretical fact that biorthogonal systems can yield better coding gain than orthonormal systems is clear, for example, from [7], and [24, Sec. 9]. One of the key papers in the literature addressing the theory of optimal biorthogonal filter banks with unconstrained filter orders is the recent work by Aas and Mullis [1]. This wonderful paper lays the foundation, and presents several useful theorems and insights. Unfortunately one of the key proofs there appears to be in error, making some of their claims inconclusive. We shall point this out more explicitly in Section $\mathrm{V}$ and in Appendix III. The purpose of our paper is to formulate the main problem and provide insights by considering a number of special cases. However, the optimal biorthogonal SBC problem in its general form is still open. One of the goals of this paper is to present the current state of research on this topic.

\section{A. Paper Outline}

All results are for the maximally decimated uniform filter bank (equal decimation $M$ in all $M$ subbands) with unconstrained filter orders. We first formulate the biorthogonal optimization problem in Section II, and in Section III we review the recent results on the orthonormal case from [24]. In Sections IV-VII we consider the biorthogonal case. Section IV shows that the biorthogonal problem can be decou-

\footnotetext{
${ }^{1}$ With a slightly different set of assumptions, it can be shown in a less involved manner that the best $\mathbf{T}$ is indeed unitary, e.g., see [24].
} 
pled into an orthonormal part and a remainder in some sense. It shows, in particular, that the biorthogonal case can be solved in its generality if it can be solved for diagonal power spectrum matrices. In the context of optimality, this section also reviews a special case called the prefiltered orthonormal filter bank. Section V develops bounds on the reconstruction error in the biorthogonal case. Restricted special cases of the biorthogonal system are then considered in Section VI; for these, the bounds reduce to a simpler form which can be achieved by proper choice of the filter matrices. Section VII provides a different performance bound based on the determinant of the psd matrix, and is sometimes useful to gain insights; for example, it can be used to find the class of inputs for which the best orthonormal system is as good as the best biorthogonal system (Corollary 2). It will also be used to show (Theorem 3) that a restricted biorthogonal system called the prefiltered orthonormal filter bank (Fig. 11) is sufficiently general for some restricted power spectra such as the one demonstrated later in Fig. 13(a). The present status and open problems will be summarized in Section VIII.

\section{B. Definitions and Notations}

Bold faced quantities represent matrices and vectors. The notations $\mathbf{A}^{T}, \mathbf{A}^{*}$, and $\mathbf{A}^{\dagger}$ represent, respectively, the transpose, conjugate, and transpose-conjugate of $\mathbf{A}$. The notation $\|\mathbf{v}\|$ indicates the vector norm, that is, $\|\mathbf{v}\|^{2}=\mathbf{v}^{\dagger} \mathbf{v}$. The filter bank in Fig. 1 is said to be biorthogonal if $\mathbf{R}\left(e^{j \omega}\right) \mathbf{E}\left(e^{j \omega}\right)=\mathbf{I}$ and orthonormal if $\mathbf{E}\left(e^{j \omega}\right)$ is paraunitary, i.e., unitary for all $\omega$. In the latter case, biorthogonality is achieved by setting $\mathbf{R}\left(e^{j \omega}\right)=\mathbf{E}^{\dagger}\left(e^{j \omega}\right)$ or, equivalently, $F_{i}\left(e^{j \omega}\right)=H_{i}^{*}\left(e^{j \omega}\right)$. Since filters are allowed to be ideal, their $z$-transforms do not necessarily exist, and $z$ should be regarded as an abbreviation for $e^{j \omega}$. For example, $P(z)$ in Fig. 11 is not a $z$-transform, but a notation for $P\left(e^{j \omega}\right)$.

\section{The PROBlem Formulation}

All the results of this paper are for maximally decimated uniform filter banks (identical decimation ratio $M$ in all $M$ subbands) and unconstrained filter orders. The part of Fig. 1(b) involved in the optimization process is shown separately in Fig. 2. Here the vector process $\mathrm{x}(n)$ is the blocked version of the scalar process $x(n)$ of Fig. 1. We assume that the vector process $\mathrm{x}(n)$ is wide sense stationary (WSS). The power spectra (psd matrices) of the WSS vectors $\mathbf{x}(n)$ and $\mathbf{y}(n)$ are denoted, respectively, by $\mathbf{S}_{x x}\left(e^{j \omega}\right)$ and $\mathbf{S}_{y y}\left(e^{j \omega}\right)$, so that $\mathbf{S}_{y y}\left(e^{j \omega}\right)=\mathbf{E}\left(e^{j \omega}\right) \mathbf{S}_{x x}\left(e^{j \omega}\right) \mathbf{E}^{\dagger}\left(e^{j \omega}\right)$. When the scalar input process $x(n)$ in Fig. 1 is WSS we can define its power spectrum $S_{x x}\left(e^{j \omega}\right)$, which should be carefully distinguished from the psd matrix $\mathbf{S}_{x x}\left(e^{j \omega}\right)$ of the vector process $\mathrm{x}(n)$.

We will find in the sequel that the eigenvalues of $\mathbf{S}_{x x}\left(e^{j \omega}\right)$ appear in various denominators. To make this meaningful, we will assume throughout that $\mathbf{S}_{x x}\left(e^{j \omega}\right)$ is nonsingular. The output WSS process $\mathbf{y}(n)$ in Fig. 2 is quantized componentwise by uniform scalar quantizers which are modeled as additive noise sources $q_{i}(n)$. The quantizer error vector is $\mathbf{q}(n)=\hat{\mathbf{y}}(n)-\mathbf{y}(n)$ and the reconstruction error vector is $\mathbf{e}(n)=\hat{\mathbf{x}}(n)-\mathbf{x}(n)$. The error vector $\mathbf{e}(n)$ can be regarded

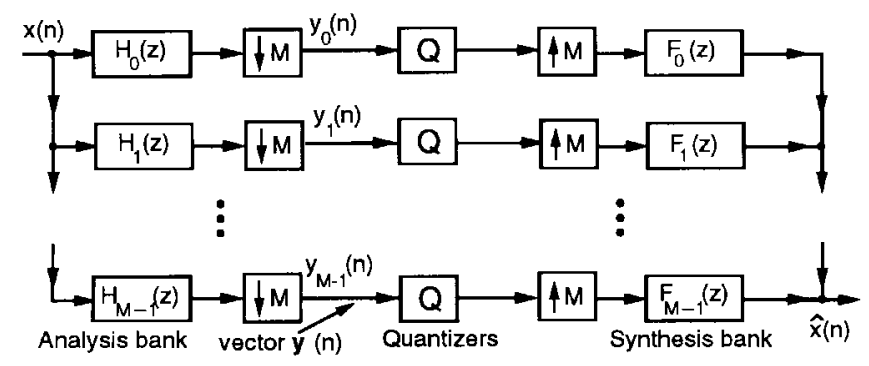

(a)

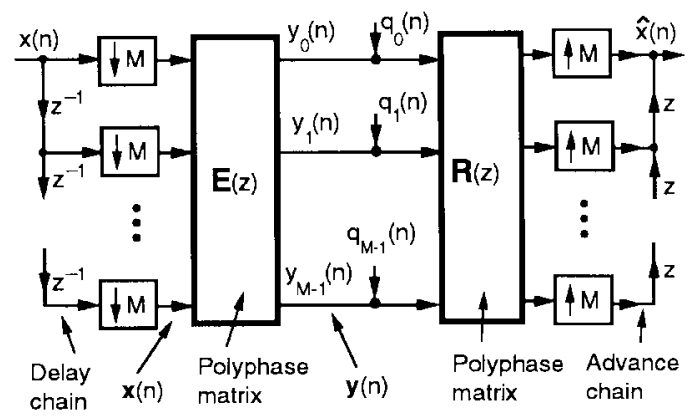

(b)

Fig. 1. (a) The maximally decimated subband coder schematic and (b) the polyphase representation.

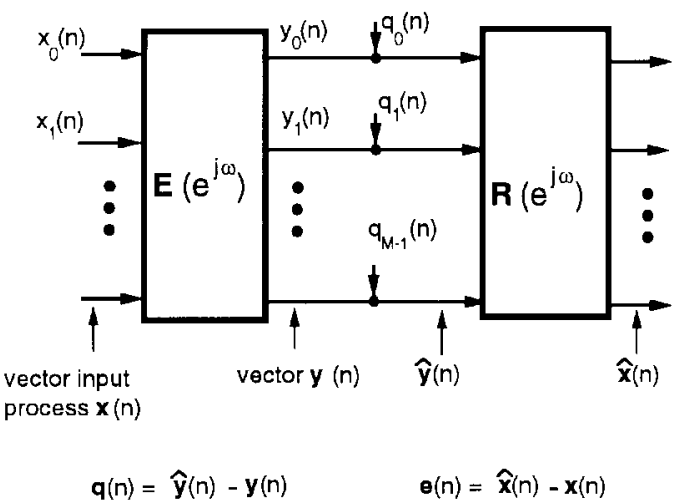

Fig. 2. The formulation of the optimal biorthogonal subband coder problem.

as the output of the synthesis polyphase matrix $\mathbf{R}\left(e^{j \omega}\right)$ in response to the quantizer error process $\mathbf{q}(n)$. The noise sources $q_{i}(n)$ are assumed to be zero-mean, uncorrelated, and white with variances $\sigma_{q_{i}}^{2}=c 2^{-2 b_{i}} \sigma_{y_{i}}^{2}$ where $b_{i}$ is the number of bits assigned to the $i$ th subband quantizer. ${ }^{2}$ Assuming optimal bit allocation with fixed bit rate $b=\sum_{i} b_{i} / M$, it can be shown (Appendix 1) that the mean square reconstruction error is

$$
\mathcal{E} \triangleq E\left[\mathbf{e}^{\dagger}(n) \mathbf{e}(n)\right]=c M 2^{-2 b} \phi^{1 / M}
$$

where

$$
\begin{aligned}
\phi \triangleq & \prod_{i=0}^{M-1} \int_{0}^{2 \pi}\left(\mathbf{E}\left(e^{j \omega}\right) \mathbf{S}_{x x}\left(e^{j \omega}\right) \mathbf{E}^{\dagger}\left(e^{j \omega}\right)\right)_{i i} d \omega / 2 \pi \\
& \times \int_{0}^{2 \pi}\left(\mathbf{R}^{\dagger}\left(e^{j \omega}\right) \mathbf{R}\left(e^{j \omega}\right)\right)_{i i} d \omega / 2 \pi
\end{aligned}
$$

\footnotetext{
${ }^{2}$ This is a standard assumption made in most of subband and transform coding theory. Its origin is explained in [11] and [23]. It is strictly justified only in the high bit rate case. The constant $c$, which depends on the nature of the statistics of the quantizer input [11], [23], is assumed to be the same for all subbands.
} 
The aim therefore is to choose $\mathbf{E}\left(e^{j \omega}\right)$ and $\mathbf{R}\left(e^{j \omega}\right)$ subject to the biorthogonality constraint $\mathbf{R}\left(e^{j \omega}\right) \mathbf{E}\left(e^{j \omega}\right)=\mathbf{I}$, such that the objective function $\phi$ is minimized. Throughout this paper, this is the meaning of "optimality." For the orthonormal case $\mathbf{R}^{\dagger}\left(e^{j \omega}\right) \mathbf{R}\left(e^{j \omega}\right)=\mathbf{I}$, so that

$$
\begin{aligned}
\phi & =\prod_{i=0}^{M-1} \int_{0}^{2 \pi}\left(\mathbf{E}\left(e^{j \omega}\right) \mathbf{S}_{x x}\left(e^{j \omega}\right) \mathbf{E}^{\dagger}\left(e^{j \omega}\right)\right)_{i i} d \omega / 2 \pi \\
& =\prod_{i=0}^{M-1} \sigma_{y_{i}}^{2} \quad \text { (orthonormal case) }
\end{aligned}
$$

where $\sigma_{y_{i}}^{2}$ is the variance of the $i$ th component $y_{i}(n)$ of the transformed vector $\mathbf{y}(n)$. Thus, for the orthonormal case, the optimization is equivalent to minimizing the product of the transformed variances $\sigma_{y_{i}}^{2}$. A special case worth noting is $\mathbf{E}\left(e^{j \omega}\right)=\mathbf{I}$, whence $\phi=\prod_{i} \sigma_{x_{i}}^{2}$ where $\sigma_{x_{i}}^{2}$ is the variance of the $i$ th component of $\mathrm{x}(n)$. In this case $\mathcal{E}=$ $c M 2^{-2 b}\left(\prod_{i} \sigma_{x_{i}}^{2}\right)^{1 / M}$, and is accomplished by optimal bit allocation alone (Appendix I).

The formulation of Fig. 2 involves only the vector process $\mathbf{x}(n)$ and the transform matrix $\mathbf{E}\left(e^{j \omega}\right)$ which is like a "transform coder with memory." For the usual special case where the vector $\mathrm{x}(n)$ is a blocked version of a scalar signal $x(n)$ we obtain the subband coder of Fig. 1(b), whose reconstruction error $e(n)=\hat{x}(n)-x(n)$ is the interleaved version of the errors at the output of $\mathbf{R}\left(e^{j \omega}\right)$ in Fig. 2. So the variance of $e(n)$ is periodic- $M$, and the average reconstruction error is

$$
\mathcal{E}_{\mathrm{SBC}}=\frac{1}{M} \sum_{n=0}^{M-1} E\left[|\hat{x}(n)-x(n)|^{2}\right]=\frac{1}{M} E\left[\mathbf{e}^{\dagger}(n) \mathbf{e}(n)\right] .
$$

If this is minimized we have the optimum biorthogonal subband coder. So optimization of $\mathbf{E}\left(e^{j \omega}\right)$ for minimizing the mean square error in Fig. 2 is identical to the optimization of the biorthogonal subband coder in Fig. 1. We shall therefore refer to Fig. 2 as the biorthogonal subband coder (orthonormal when $\mathbf{E}\left(e^{j \omega}\right)$ is unitary), even though the WSS process $\mathrm{x}(n)$ may not be the blocked version of a scalar WSS process $x(n)$.

The WSS property of the vector $\mathrm{x}(n)$ is equivalent to the property that the scalar signal $x(n)$ is cyclo-WSS [17] with period $M$. This is more general than assuming that $x(n)$ itself is WSS. For the special case where $x(n)$ is WSS, we can define a power spectrum $S_{x x}\left(e^{j \omega}\right)$ and variance $\sigma_{x}^{2}$ for it, and express the performance in terms of the coding gain. This is defined by comparing $\mathcal{E}_{\mathrm{SBC}}$ with the mean square value $\mathcal{E}_{\text {direct }}$ of the direct quantization error (roundoff quantizer) having the same bit rate $b$. That is, $G_{\mathrm{SBC}}(M)=\mathcal{E}_{\text {direct }} / \mathcal{E}_{\mathrm{SBC}}$.

When $x(n)$ is WSS, the quantity $\int\left(\mathbf{E}\left(e^{j \omega}\right) \mathbf{S}_{x x}\left(e^{j \omega}\right) \mathbf{E}^{\dagger}\right.$ $\left.\left(e^{j \omega}\right)\right)_{i i} d \omega / 2 \pi$ in (2), which is the subband variance in Fig. 1(a), can be written as $\int S_{x x}\left(e^{j \omega}\right)\left|H_{i}\left(e^{j \omega}\right)\right|^{2} d \omega / 2 \pi$. Similarly it can be verified that $\int\left(\mathbf{R}^{\dagger}\left(e^{j \omega}\right) \mathbf{R}\left(e^{j \omega}\right)\right)_{i i} d \omega / 2 \pi$ $=\int\left|F_{i}\left(e^{j \omega}\right)\right|^{2} d \omega / 2 \pi$, which is the energy of the $i$ th synthesis filter. Thus $\phi=\prod_{i=0}^{M-1} \int_{0}^{2 \pi} S_{x x}\left(e^{j \omega}\right)\left|H_{i}\left(e^{j \omega}\right)\right|^{2} d \omega / 2 \pi \int_{0}^{2 \pi} \mid$ $\left.F_{i}\left(e^{j \omega}\right)\right|^{2} d \omega / 2 \pi$ in this case. For the biorthogonal filter bank with optimal bit allocation the coding gain can be expressed [7] as in (5), shown at the bottom of the page. For the orthonormal case this reduces to $G_{\mathrm{SBC}}(M)=\sigma_{x}^{2} /\left(\prod_{i=0}^{M-1} \sigma_{y_{i}}^{2}\right)^{1 / M}$. Maximization of coding gain is equivalent to minimizing the mean square error (1), or equivalently, minimizing the quantity $\phi$ in (2). Using orthonormality we can write $M \sigma_{x}^{2}=\sum_{i=0}^{M-1} \sigma_{y_{i}}^{2}$, so the coding gain becomes the ratio of the arithmetic to geometric mean of the subband variances $\sigma_{y_{i}}^{2}$.

\section{REVIEW OF THE ORTHONORMAL CASE}

In orthogonal transform coding theory (where $\mathbf{E}\left(e^{j \omega}\right)$ in Fig. 2 is a constant unitary matrix $\mathbf{T})$ it is well known that the transform is optimal if and only if $E\left[y_{i}(n) y_{k}^{*}(n)\right]=0$ for $i \neq k$, and for all $n$. For orthonormal subband coders where $\mathbf{E}\left(e^{j \omega}\right)$ is a unitary function of $\omega$, the optimality conditions have recently been derived [24], [25], and are briefly reviewed here. All detailed proofs can be found in [24].

First, for an orthonormal subband coder to be optimal, it is necessary that the decimated subband random processes be uncorrelated, that is,

$$
E\left[y_{i}(n) y_{k}^{*}(m)\right]=0
$$

for $i \neq k$, and for all $n, m$. This condition will also be referred to as total decorrelation of subbands. Equivalently, the power spectrum matrix of the vector process $\mathbf{y}(n)$ must be diagonal:

$$
\mathbf{S}_{\mathbf{y y}}\left(e^{j \omega}\right)=\left[\begin{array}{cccc}
S_{0}\left(e^{j \omega}\right) & 0 & \cdots & 0 \\
0 & S_{1}\left(e^{j \omega}\right) & \cdots & 0 \\
\vdots & \vdots & \ddots & \vdots \\
0 & 0 & \cdots & S_{M-1}\left(e^{j \omega}\right)
\end{array}\right]
$$

where $S_{i}\left(e^{j \omega}\right)$ is the psd of $y_{i}(n)$. This result is proved by contradiction: thus, suppose $E\left[y_{0}(n) y_{1}^{*}(n-k)\right] \neq 0$ for some $k$ (and for all $n$, by the WSS property). We can then decrease the reconstruction error without violating orthonormality: for this, use a delay $z^{-k}$ and a unitary matrix $\Theta$ to transform the pair $\left\{y_{0}(n), y_{1}(n-k)\right\}$ into an uncorrelated pair $\left\{w_{0}(n), w_{1}(n)\right\}$ (Fig. 3). This can be done by choosing $\Theta$ to be the KLT matrix for the vector WSS process $\left[y_{0}(n) y_{1}(n-k)\right]^{T}$. The modified polyphase matrix continues to be paraunitary, i.e., the filter bank is still orthonormal since $\Theta$ is unitary. It can be verified, however, that $\sigma_{w_{0}}^{2} \sigma_{w_{1}}^{2}<\sigma_{y_{0}}^{2} \sigma_{y_{1}}^{2}$, so the objective function $\phi$ in (3) is decreased.

For optimality in the orthonormal case, it is shown in [24] that a second property called spectral majorization is also necessary. This means that the power spectra $S_{i}\left(e^{j \omega}\right)$ of $y_{i}(n)$ satisfy

$$
S_{0}\left(e^{j \omega}\right) \geq S_{1}\left(e^{j \omega}\right) \geq \cdots \geq S_{M-1}\left(e^{j \omega}\right), \quad \text { for all } \omega
$$

$$
G_{\mathrm{SBC}}(M) \triangleq \frac{\mathcal{E}_{\text {direct }}}{\mathcal{E}_{\mathrm{SBC}}}=\sigma_{x}^{2} / \phi^{1 / M}=\frac{\sigma_{x}^{2}}{\left(\prod_{i=0}^{M-1} \int_{0}^{2 \pi} S_{x x}\left(e^{j \omega}\right)\left|H_{i}\left(e^{j \omega}\right)\right|^{2} d \omega / 2 \pi \int_{0}^{2 \pi}\left|F_{i}\left(e^{j \omega}\right)\right|^{2} d \omega / 2 \pi\right)^{1 / M}}
$$




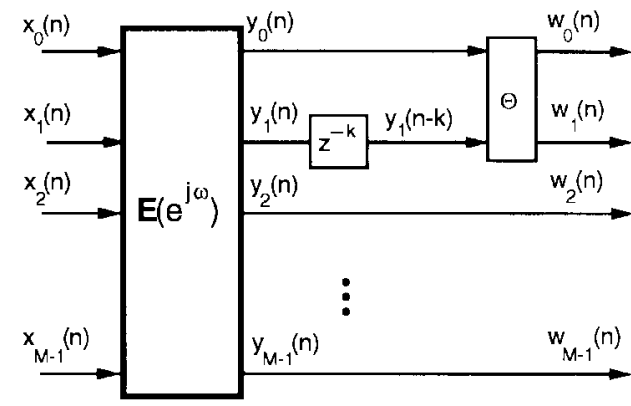

Fig. 3. Proof that total decorrelation is necessary.

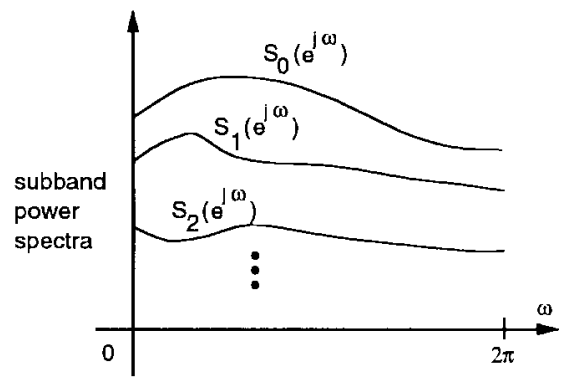

Fig. 4. Example of the spectral majorization property.

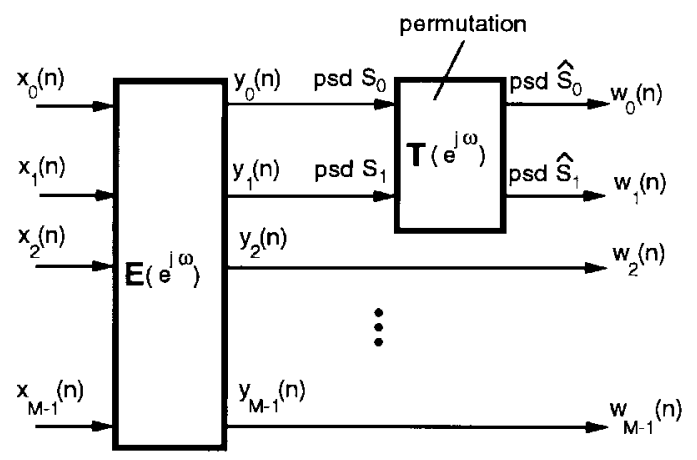

Fig. 5. Proof that spectral majorization is necessary.

(assuming the ordering to be such that $\sigma_{y_{0}}^{2} \geq \sigma_{y_{1}}^{2} \geq \cdots$ ). This is demonstrated in Fig. 4. The reason is that if majorization is not satisfied, the objective function $\phi$ can be decreased further without violating orthonormality. For example, assume $S_{0}\left(e^{j \omega}\right)>S_{1}\left(e^{j \omega}\right)$ for some values of $\omega$ and $S_{1}\left(e^{j \omega}\right)>$ $S_{0}\left(e^{j \omega}\right)$ for some other values of $\omega$. We can then cascade a frequency dependent permutation matrix $\mathbf{T}\left(e^{j \omega}\right)$ (which is a paraunitary LTI system) as shown in Fig. 5 such that the new pair of power spectra $\hat{S}_{0}\left(e^{j \omega}\right), \hat{S}_{1}\left(e^{j \omega}\right)$ satisfies $\hat{S}_{0}\left(e^{j \omega}\right) \geq$ $\hat{S}_{1}\left(e^{j \omega}\right)$ for all $\omega$. Moreover, for each $\omega, \hat{S}_{0}\left(e^{j \omega}\right) \geq S_{0}\left(e^{j \omega}\right)$ whereas $\hat{S}_{1}\left(e^{j \omega}\right) \leq S_{1}\left(e^{j \omega}\right)$. Thus, the variances of the new signals $w_{0}(n)$ and $w_{1}(n)$ are such that $\sigma_{w_{0}}^{2} \geq \sigma_{y_{0}}^{2}$ and $\sigma_{w_{1}}^{2} \leq \sigma_{y_{1}}^{2}$. Since $\mathbf{T}\left(e^{j \omega}\right)$ is paraunitary by construction, the filter bank remains orthonormal, and, in particular $\sigma_{w_{0}}^{2}+\sigma_{w_{1}}^{2}=$ $\sigma_{y_{0}}^{2}+\sigma_{y_{1}}^{2}$. From this it can be verified that $\sigma_{w_{0}}^{2} \sigma_{w_{1}}^{2}<\sigma_{y_{0}}^{2} \sigma_{y_{1}}^{2}$, so the objective function $\phi$ has been decreased.

Any orthonormal filter bank with nonoverlapping analysis filters satisfies total decorrelation. On the other hand, the delay chain filter bank (Fig. 1(a) with $H_{k}(z)=z^{-k}, F_{k}(z)=$ $\left.z^{k}\right)$ satisfies spectral majorization (all $S_{k}\left(e^{j \omega}\right)$ are identical).

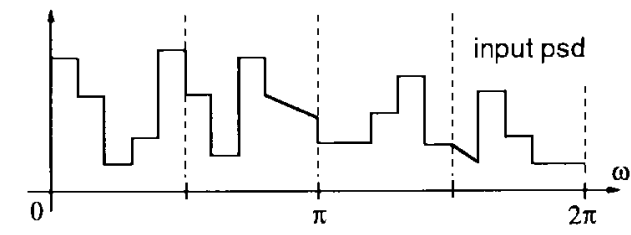

(a)

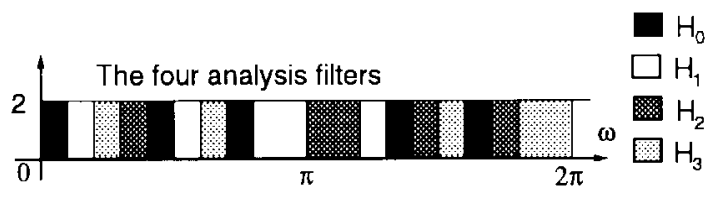

(b)

Fig. 6. (a) An input psd and (b) the four-band optimal orthonormal filter bank.

Thus, neither total decorrelation, nor spectral majorization is sufficient for optimality of orthonormal subband coders. However, the following has been proved in [24].

Theorem 1-Optimal Orthonormal Subband Coder: For the orthonormal case, where $\mathbf{E}\left(e^{j \omega}\right)$ is restricted to be unitary for all $\omega$, total decorrelation and spectral majorization of the transformed signal $\mathbf{y}(n)$ are together necessary and sufficient for the minimization of $\phi$ [i.e., minimization of the reconstruction error $\mathcal{E}$ in (1)].

To see this, note that since $\mathbf{E}\left(e^{j \omega}\right)$ is unitary, the diagonalized psd elements $S_{i}\left(e^{j \omega}\right)$ are the eigenvalues of the psd matrix $\mathbf{S}_{x x}\left(e^{j \omega}\right)$ of the vector process $\mathbf{x}(n)$ (Fig. 2). (The columns of $\mathbf{E}^{\dagger}\left(e^{j \omega}\right)$ are the corresponding eigenvectors.) The majorization property imposes an ordering among these eigenvalues. The majorized and decorrelated set of power spectra $\left\{S_{i}\left(e^{j \omega}\right)\right\}$ is therefore unique, since the ordered set of eigenvalues is unique. Since majorization and decorrelation are necessary and since there is only one set of such subband power spectra, it follows that these two conditions together imply optimality.

It can be shown [24] that majorization and decorrelation can together be satisfied by designing the orthonormal analysis bank in Fig. 1(a) using ideal nonoverlapping filters with appropriately chosen passbands. Fig. 6 shows the example of an input psd and the four-channel optimal orthonormal filter bank. Each filter in this system has a total passband width $2 \pi / M$ (and passband response $\sqrt{M}$ ), and provides an aliasfree (though multiband) support for decimation by $M$. In fact, these optimal designs can be constructed by designing the analysis filters $H_{i}\left(e^{j \omega}\right)$ sequentially, i.e., one at a time. For this, each filter $H_{i}\left(e^{j \omega}\right)$ is chosen to be an energy compaction filter (see [24]) for an appropriate psd derived from $S_{x x}\left(e^{j \omega}\right)$.

Majorized-Eigenvalue Notation: The elements $S_{i}\left(e^{j \omega}\right)$ of the diagonal matrix $\mathbf{S}_{y y}\left(e^{j \omega}\right)$ are the eigenvalues of $\mathbf{S}_{x x}\left(e^{j \omega}\right)$. Since these also satisfy the majorization property in the optimal case, we will reserve a particular notation for these, namely $\eta_{i}\left(e^{j \omega}\right)$. Summarizing, $\left\{\eta_{i}\left(e^{j \omega}\right)\right\}$ is the set of eigenvalues of the psd matrix $\mathbf{S}_{x x}\left(e^{j \omega}\right)$ ordered such that $\eta_{i}\left(e^{j \omega}\right) \geq$ $\eta_{i+1}\left(e^{j \omega}\right)$. It is also the set of decimated power spectra for the optimal orthonormal subband coder.

The quantity $\phi$ for the optimal orthonormal subband coder can be derived from (3) by noticing that $\sigma_{y_{i}}^{2}$ are the variances 
corresponding to the power spectra $\eta_{i}\left(e^{j \omega}\right)$. Thus,

$$
\phi_{\mathrm{opt}}=\prod_{i=0}^{M-1} \int_{0}^{2 \pi} \eta_{i}\left(e^{j \omega}\right) d \omega / 2 \pi \quad \text { (orthonormal case) }
$$

and the coding gain of the optimal orthonormal subband coder is

$$
G_{\perp}(M)=\frac{\sigma_{x}^{2}}{\left(\prod_{i=0}^{M-1} \int_{0}^{2 \pi} \eta_{i}\left(e^{j \omega}\right) d \omega / 2 \pi\right)^{1 / M}} .
$$

\section{A. More on Spectral Majorization}

The above results for orthonormal subband coders imply a linear-algebraic inequality, which we shall find useful later for the optimization of biorthogonal subband coders; this was also observed in [1] where the proof was purely algebraic, based on theorems in matrix theory.

Corollary 1-A Matrix Inequality: Let $\mathbf{P}\left(e^{j \omega}\right)$ be an $M \times M$ Hermitian positive definite matrix with eigenvalues $\eta_{i}\left(e^{j \omega}\right)$, ordered such that $\eta_{0}\left(e^{j \omega}\right) \geq \eta_{1}\left(e^{j \omega}\right) \geq$ $\cdots \eta_{M-1}\left(e^{j \omega}\right)$ for all $\omega$. Then

$$
\prod_{i=0}^{M-1} \int_{0}^{2 \pi}\left[\mathbf{P}\left(e^{j \omega}\right)\right]_{i i} d \omega / 2 \pi \geq \prod_{i=0}^{M-1} \int_{0}^{2 \pi} \eta_{i}\left(e^{j \omega}\right) d \omega / 2 \pi .
$$

Proof: Without loss of generality, assume that $\mathbf{P}\left(e^{j \omega}\right)$ is the psd matrix of a zero-mean WSS vector process $\mathrm{x}(n)$. Then $E\left[\mathbf{x}(n) \mathbf{x}^{\dagger}(n)\right]=\int_{0}^{2 \pi} \mathbf{P}\left(e^{j \omega}\right) d \omega / 2 \pi$. Thus the left hand side in (11) is the product $\prod_{i} \sigma_{x_{i}}^{2}$, where $\sigma_{x_{i}}^{2}$ is the variance of the $i$ th component of $\mathrm{x}(n)$. Suppose we pass $\mathrm{x}(n)$ through a paraunitary transfer matrix $\mathbf{E}\left(e^{j \omega}\right)$ which performs total decorrelation as well as spectral majorization. Then $\eta_{i}\left(e^{j \omega}\right)$ is the psd of the $i$ th element of the output process $\mathbf{y}(n)$. Thus the right-hand side in (11) is the product $\prod_{i} \sigma_{y_{i}}^{2}$ of the variances of the output components $y_{i}(n)$. Since the above choice of $\mathbf{E}\left(e^{j \omega}\right)$ reperesents an optimal orthonormal subband coder (Theorem 1), we have $\prod_{i} \sigma_{x_{i}}^{2} \geq \prod_{i} \sigma_{y_{i}}^{2}$, proving (11). $\square$

Majorized Version of a Set: Given a set of nonnegative functions $\alpha_{i}\left(e^{j \omega}\right), 0 \leq i \leq M-1$, define a diagonal matrix $\boldsymbol{\Lambda}_{\alpha}\left(e^{j \omega}\right)$ with diagonal elements $\alpha_{i}\left(e^{j \omega}\right)$. Let $\mathbf{T}\left(e^{j \omega}\right)$ be a frequency dependent permutation matrix (in particular, it is paraunitary), and define $\boldsymbol{\Lambda}_{\beta}\left(e^{j \omega}\right)=\mathbf{T}\left(e^{j \omega}\right) \boldsymbol{\Lambda}_{\alpha}\left(e^{j \omega}\right) \mathbf{T}^{\dagger}\left(e^{j \omega}\right)$. Then $\boldsymbol{\Lambda}_{\beta}\left(e^{j \omega}\right)$ is also a diagonal matrix with nonnegative elements. We can always choose the permutation $\mathbf{T}\left(e^{j \omega}\right)$ such that the diagonal elements of $\boldsymbol{\Lambda}_{\beta}\left(e^{j \omega}\right)$ satisfy the majorization property $\beta_{i}\left(e^{j \omega}\right) \geq \beta_{i+1}\left(e^{j \omega}\right)$. It follows from Corollary 1 that

$$
\prod_{i=0}^{M-1} \int_{0}^{2 \pi} \alpha_{i}\left(e^{j \omega}\right) d \omega / 2 \pi \geq \prod_{i=0}^{M-1} \int_{0}^{2 \pi} \beta_{i}\left(e^{j \omega}\right) d \omega / 2 \pi .
$$

We can regard $\boldsymbol{\Lambda}_{\alpha}\left(e^{j \omega}\right)$ to be the psd matrix of a vector process $\mathbf{x}_{\alpha}(n)$ with uncorrelated components. Then $\boldsymbol{\Lambda}_{\beta}\left(e^{j \omega}\right)$ is the psd matrix of the output $\mathbf{x}_{\beta}(n)$ of $\mathbf{T}\left(e^{j \omega}\right)$, in response to the input $\mathrm{x}_{\alpha}(n)$. We say that $\left\{\beta_{i}\left(e^{j \omega}\right)\right\}$ is the majorized version of the set $\left\{\alpha_{i}\left(e^{j \omega}\right)\right\}$ [equivalently, the process $\mathbf{x}_{\beta}(n)$ is the majorized version of the process $\left.\mathrm{x}_{\alpha}(n)\right]$.

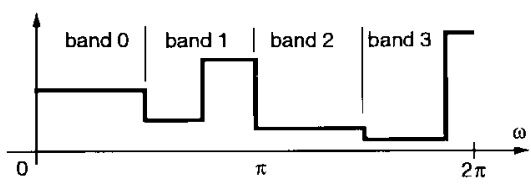

(a)

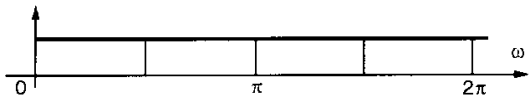

(b)

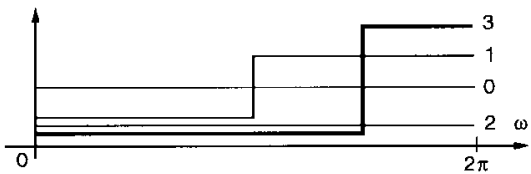

(c)

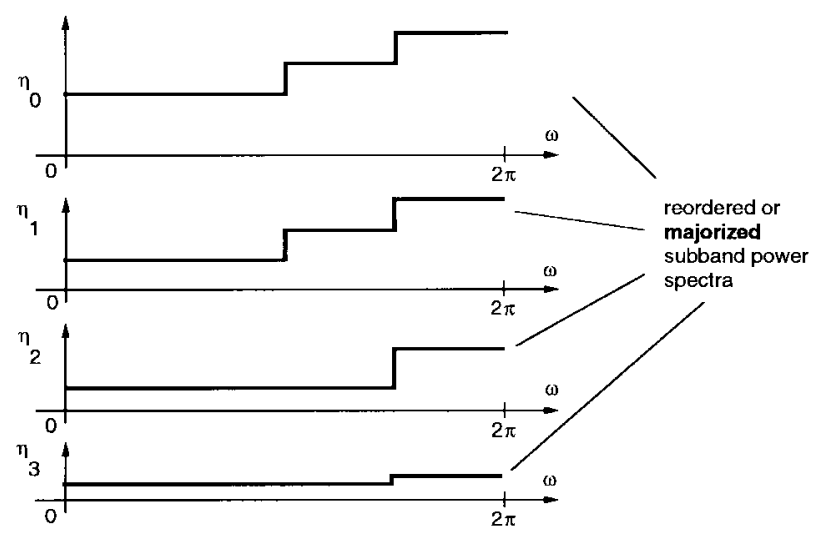

(d)

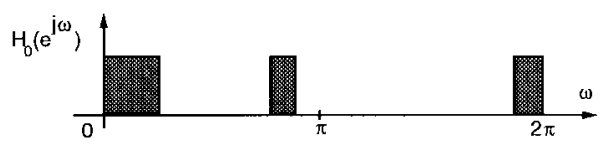

(e)

Fig. 7. Example of subband power spectra and ordered or majorized eigenvalues. (a) Input psd, (b) traditional stacking for four-band orthonormal subband coder, (c) decimated subband power spectra, (d) the majorized set of subband power spectra, and (e) one of the filters in an optimal orthonormal system, that is, the system which would yield the majorized subband power spectra.

\section{B. Relationship Between Majorized Eigenvalues and Input Psd}

To develop further intuition as to how $\left\{\eta_{i}\left(e^{j \omega}\right)\right\}$ are related to the psd $S_{x x}\left(e^{j \omega}\right)$ of the scalar process $x(n)$ in Fig. 1, consider the scalar psd $S_{x x}\left(e^{j \omega}\right)$ of Fig. 7(a). Fig. 7(b) shows the traditional (not optimal) ideal brickwall filters for a fourchannel subband coder. This set of nonoverlapping filters evidently performs total decorrelation. The four decimated subband signals $y_{i}(n)$ have the psd shown in Fig. 7(c). For each $\omega$, these are eigenvalues of the psd matrix $\mathbf{S}_{x x}\left(e^{j \omega}\right)$ of the blocked version $\mathrm{x}(n)$ in Fig. 1(b). Each of these four plots is a piece of $S_{x x}\left(e^{j \omega}\right)$ falling inside a filter passband, appropriately stretched by the decimation factor $M$. We can now perform frequency dependent permutations (as in Fig. 5) and reorder these eigenvalues into a majorized set. Fig. 7(d) shows these majorized power spectra which satisfy $\eta_{0}\left(e^{j \omega}\right) \geq \eta_{1}\left(e^{j \omega}\right) \geq$ $\eta_{2}\left(e^{j \omega}\right) \geq \eta_{3}\left(e^{j \omega}\right)$. It is clear that the functions $\eta_{i}\left(e^{j \omega}\right)$ are 


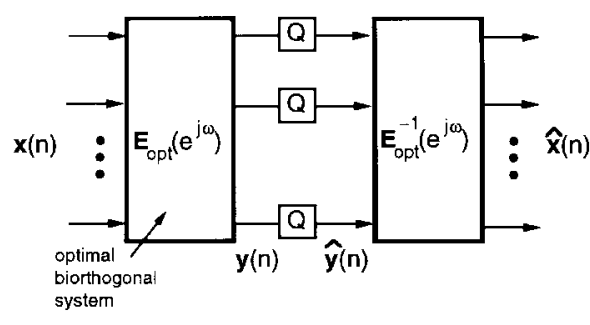

(a)

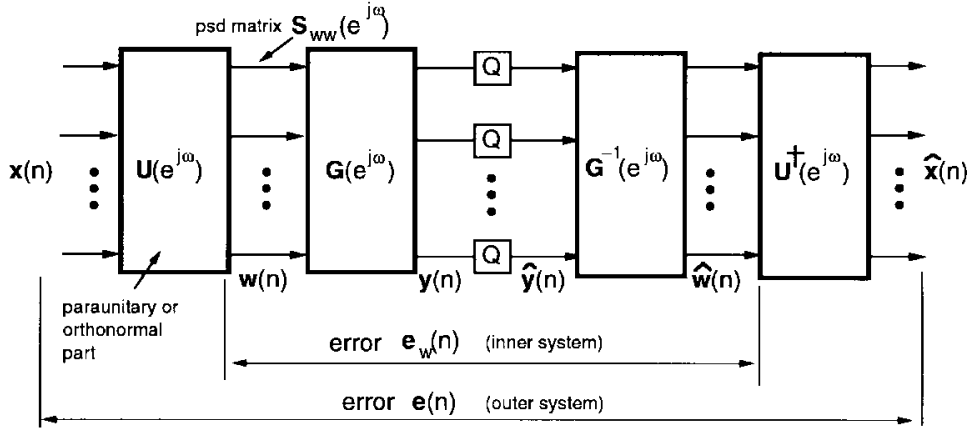

(b)

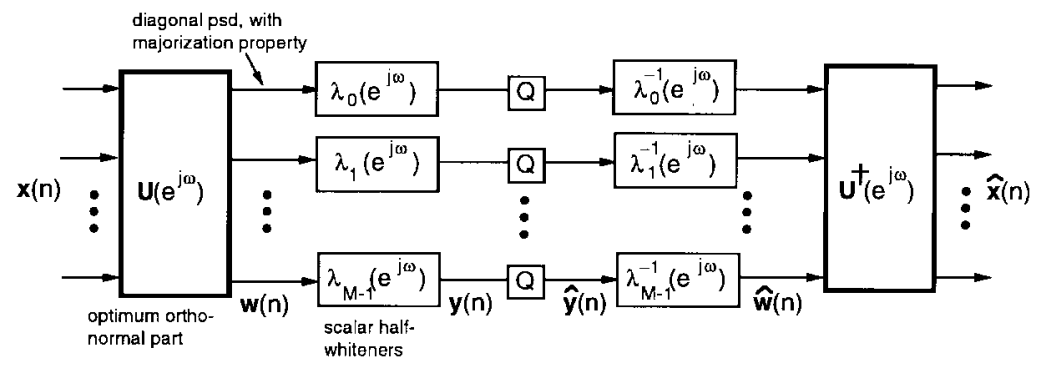

(c)

Fig. 8. (a) The optimal biorthogonal system, (b) decomposition into an orthonormal part and a remainder part, and (c) a restricted case of the biorthogonal system.

nothing but various pieces of the scalar psd $S_{x x}\left(e^{j \omega}\right)$ "pasted together in an optimal way" (after streching by $M$ ). To complete this discussion, note that though the traditional brickwall system of Fig. 7(a) performs total decorrelation, it does not in general produce a majorized set. If we take into account the subband permutations required for this, we can define the new orthonormal set of analysis filters $\left\{H_{i}\left(e^{j \omega}\right)\right\}$ which perform both total decorrelation and majorization. Fig. 7(e) shows the first filter $H_{0}\left(e^{j \omega}\right)$ of this optimum orthonormal filter bank. We leave it to the reader to figure out the remaining three filters. In general, these are ideal nonoverlapping filters tiling the frequency axis (as in Fig. 6).

On Uniqueness of the Optimal Solution: In general, the optimal solution is not unique. For example, if $x(n)$ is white then any orthonormal filter bank provides the "optimal" gain of unity. However, if the majorized eigenvalues $\left\{\eta_{i}\left(e^{j \omega}\right)\right\}$ are such that the ordering is strict, i.e., $\eta_{i}\left(e^{j \omega}\right)>\eta_{i+1}\left(e^{j \omega}\right)$ then the eigenvectors [columns of $\mathbf{E}^{\dagger}\left(e^{j \omega}\right)$ ] are unique (up to allpass scale factors). That is, two optimal solutions $\mathbf{E}_{1}\left(e^{j \omega}\right)$ and $\mathbf{E}_{2}\left(e^{j \omega}\right)$ are related as $\mathbf{E}_{2}\left(e^{j \omega}\right)=\mathcal{D}\left(e^{j \omega}\right) \mathbf{E}_{1}\left(e^{j \omega}\right)$ where $\mathcal{D}\left(e^{j \omega}\right)$ is diagonal with unit-magnitude diagonal elements. An example with unique optimal solution is the case of a strictly decreasing psd $S_{x x}\left(e^{j \omega}\right)$ (e.g., as in $1 / f$ processes). In this case the traditonal brickwall stacking Fig. 7(b) is optimal.

\section{Decoupling the Biorthogonal Problem}

In the biorthogonal case, the only constraint on the matrix $\mathbf{E}\left(e^{j \omega}\right)$ is that it be nonsingular. Our aim is to find $\mathbf{E}\left(e^{j \omega}\right)$ such that the m.s. error (1) is minimized. Suppose the solution $\mathbf{E}\left(e^{j \omega}\right)=\mathbf{E}_{\text {opt }}\left(e^{j \omega}\right)$ achieves this. Given any arbitrary paraunitary $\mathbf{U}\left(e^{j \omega}\right)$, we can always write $\mathbf{E}_{\text {opt }}\left(e^{j \omega}\right)$ in the

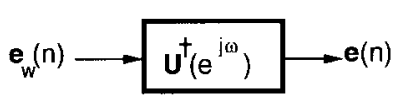

Fig. 9. Relation between the two errors.

form

$$
\mathbf{E}_{\mathrm{opt}}\left(e^{j \omega}\right)=\mathbf{G}\left(e^{j \omega}\right) \mathbf{U}\left(e^{j \omega}\right)
$$

because $\mathbf{U}\left(e^{j \omega}\right)$ is nonsingular. So the optimal biorthogonal system of Fig. 8(a) can be redrawn as in Fig. 8(b). Now consider the two reconstruction errors $\mathbf{e}(n)=\hat{\mathbf{x}}(n)-\mathbf{x}(n)$ and $\mathbf{e}_{w}(n)=\hat{\mathbf{w}}(n)-\mathbf{w}(n)$. These are related by the paraunitary matrix $\mathbf{U}\left(e^{j \omega}\right)$ as shown in Fig. 9. It therefore follows (Appendix II) that

$$
E\left[\mathbf{e}_{w}^{\dagger}(n) \mathbf{e}_{w}(n)\right]=E\left[\mathbf{e}^{\dagger}(n) \mathbf{e}(n)\right]
$$

that is, the m.s. reconstruction error for $\mathbf{w}(n)$ is the same as that for $\mathrm{x}(n)$. Of course, the choice of $\mathbf{U}\left(e^{j \omega}\right)$ will certainly affect the reconstruction error for $\mathbf{w}(n)$, but this will always be equal to the error for $\mathrm{x}(n)$.

Since the choice $\mathbf{E}_{\text {opt }}\left(e^{j \omega}\right)$ is optimal, it follows that $\mathbf{G}\left(e^{j \omega}\right)$ is optimal for its input $\mathbf{w}(n)$, i.e., it minimizes the m.s. value of $\mathbf{e}_{w}(n)$. (Otherwise, we could replace $\mathbf{G}\left(e^{j \omega}\right)$ with a better matrix $\mathbf{G}_{\text {bet }}\left(e^{j \omega}\right)$, which would make $\mathbf{G}_{\text {bet }}\left(e^{j \omega}\right) \mathbf{U}\left(e^{j \omega}\right)$ better than $\mathbf{E}_{\text {opt }}\left(e^{j \omega}\right)$, creating a conflict. $)$ This reasoning holds for any paraunitary $\mathbf{U}\left(e^{j \omega}\right)$. For example, $\mathbf{U}\left(e^{j \omega}\right)$ could be the optimal orthonormal system for $\mathbf{x}(n)$. Thus, we can always decouple the design of the optimal biorthogonal $\mathbf{E}_{\text {opt }}\left(e^{j \omega}\right)$ into two steps: 1) first design the optimal orthonormal system $\mathbf{U}\left(e^{j \omega}\right)$ which produces an output $\mathbf{w}(n)$ with the total decorrelation and spectral majorization properties, and 2) design the best biorthogonal system $\mathbf{G}\left(e^{j \omega}\right)$ for $\mathbf{w}(n)$. 


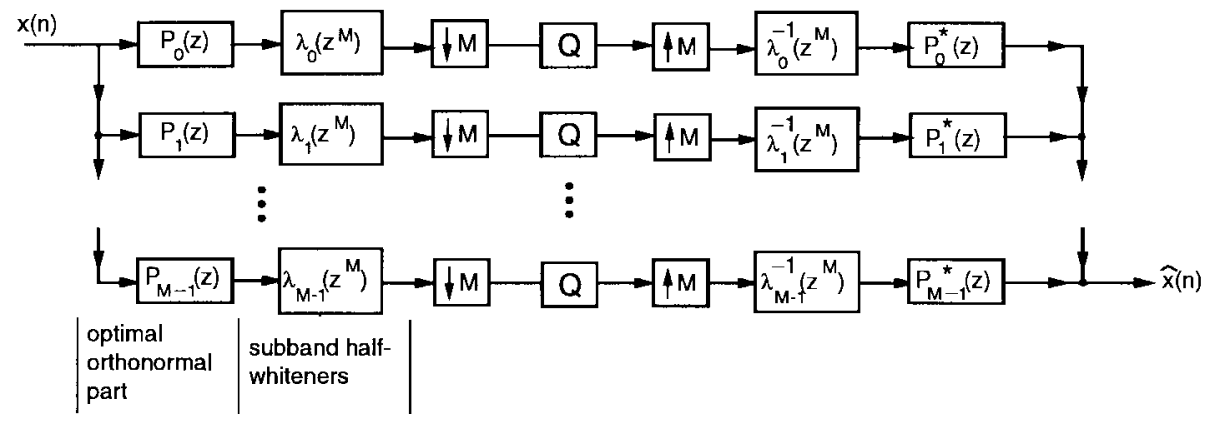

Fig. 10. The special case shown in Fig. 8(c), redrawn.

\section{A. Case of Diagonal $\mathbf{G}$}

If the components of $\mathbf{w}(n)$ are totally decorrelated, the first impression is that there is no loss of generality in restricting $\mathbf{G}\left(e^{j \omega}\right)$ to be diagonal. But we have to be careful; unless the components of $\mathbf{w}(n)$ satisfy spectral majorization, a frequency dependent permutation (paraunitary matrix) further reduces the objective function $\phi$ (Section III). So it is a loss of generality to restrict $\mathbf{G}\left(e^{j \omega}\right)$ to be diagonal.

The more interesting question therefore is: suppose the paraunitary matrix $\mathbf{U}\left(e^{j \omega}\right)$ performs total decorrelation and spectral majorization; in seeking the best biorthogonal system, is there a loss of generality in restricting $\mathbf{G}\left(e^{j \omega}\right)$ to be a diagonal matrix? Let us first find out how best we can do if we constrain $\mathbf{G}\left(e^{j \omega}\right)$ to be diagonal.

Best Diagonal G-Matrix: For diagonal $\mathbf{G}\left(e^{j \omega}\right)$, let $\lambda_{i}\left(e^{j \omega}\right)$ denote its diagonal elements. Let $S_{i}\left(e^{j \omega}\right)$ be the psd of the $i$ th component $w_{i}(n)$ of $\mathbf{w}(n)$. As in Section II we can write the m.s. value of $\mathbf{e}_{w}(n)$ as $c M 2^{-2 b} \phi_{w}^{1 / M}$ where

$$
\begin{aligned}
\phi_{w}= & \prod_{i=0}^{M-1} \int_{0}^{2 \pi}\left|\lambda_{i}\left(e^{j \omega}\right)\right|^{2} S_{i}\left(e^{j \omega}\right) d \omega / 2 \pi \\
& \times \int_{0}^{2 \pi} \frac{1}{\left|\lambda_{i}\left(e^{j \omega}\right)\right|^{2}} d \omega / 2 \pi \\
\geq & \left(\prod_{i=0}^{M-1} \int_{0}^{2 \pi} \sqrt{S_{i}\left(e^{j \omega}\right)} d \omega / 2 \pi\right)^{2}
\end{aligned}
$$

using the Cauchy-Schwartz inequality. Equality is achieved if

$$
\lambda_{i}\left(e^{j \omega}\right)=1 / S_{i}^{1 / 4}\left(e^{j \omega}\right)
$$

which is the half-whitening filter [11], [23]. If we assume that $\mathbf{U}\left(e^{j \omega}\right)$ has performed total decorrelation, then $S_{i}\left(e^{j \omega}\right)$ are the eigenvalues of the psd matrix $\mathbf{S}_{x x}\left(e^{j \omega}\right)$. If we further assume that $\mathbf{U}\left(e^{j \omega}\right)$ also performs spectral majorization, then $S_{i}\left(e^{j \omega}\right)$ are the ordered eigenvalues $\eta_{i}\left(e^{j \omega}\right)$ (in the notation of Section III). So here is the summary of what we have shown.

Lemma 1: In Fig. 8(c), suppose $\mathbf{U}\left(e^{j \omega}\right)$ is the optimum orthonormal solution for $\mathrm{x}(n)$, and $\lambda_{i}\left(e^{j \omega}\right)$ are the optimal (half-whitening) filters for their respective inputs. Then the objective function $\phi$ has the value

$$
\phi_{\eta} \triangleq\left(\prod_{i=0}^{M-1} \int_{0}^{2 \pi} \sqrt{\eta_{i}\left(e^{j \omega}\right)} d \omega / 2 \pi\right)^{2}
$$

where $\eta_{i}\left(e^{j \omega}\right)$ are the majorized eigenvalues (Section III) of the input psd matrix $\mathbf{S}_{x x}\left(e^{j \omega}\right)$.

The quantity $\phi_{\eta}$ will play a significant role in all our discussions. It depends only on the input psd matrix $\mathbf{S}_{x x}\left(e^{j \omega}\right)$, since $\left\{\eta_{i}\left(e^{j \omega}\right)\right\}$ is the unique set of ordered eigenvalues of $\mathbf{S}_{x x}\left(e^{j \omega}\right)$. In fact at the end of Section III we showed that each $\eta_{i}\left(e^{j \omega}\right)$ is obtained by pasting together appropriate pieces of the scalar psd $S_{x x}\left(e^{j \omega}\right)$ (after $M$-fold stretch due to decimation).

It is clear that the special form of biorthogonal system shown in Fig. 8(c) achieves $\phi=\phi_{\eta}$. An open problem here is to prove (or disprove, say, by a counterexample) that the optimal biorthogonal system satisfies $\phi_{\mathrm{opt}}=\phi_{\eta}$ or, equivalently, that

$$
\phi \geq \phi_{\eta}
$$

for any biorthogonal filter bank. If this is the case, then the optimal biorthogonal system can be represented as in Fig. 8(c). While this claim has been made implicitly in [1], an error in the proof there makes it inconclusive [15]. This will be clear from Section V-A here; readers familiar with [1] can also see Appendix III.

\section{B. The Prefiltered Orthonormal Filter Bank}

The special biorthogonal system shown in Fig. 8(c) can be redrawn in terms of the original subband coder by inserting the delay chains, decimators, and expanders as in Fig. 1(b). The result can then be redrawn as in Fig. 10 where the filters $P_{i}\left(e^{j \omega}\right)$ are the analysis filters of the orthonormal SBC corresponding to the polyphase matrix $\mathbf{U}\left(e^{j \omega}\right)$. The halfwhitening filters have been moved past the decimator and expander using noble identities [23].

Since $\left\{P_{i}\left(e^{j \omega}\right)\right\}$ is an optimal orthonormal set, the filters $P_{i}\left(e^{j \omega}\right)$ can be assumed to be ideal nonoverlapping filters (with passband response $\sqrt{M}$ ) without loss of generality [24] as demonstrated earlier in Fig. 6(b). The filters $\lambda_{i}\left(e^{j \omega M}\right)$ following $P_{i}\left(e^{j \omega}\right)$ can therefore be replaced with

$$
\hat{\lambda}_{i}\left(e^{j \omega}\right)= \begin{cases}\lambda_{i}\left(e^{j \omega M}\right), & \text { in the passband of } P_{i}\left(e^{j \omega}\right) \\ 0, & \text { elsewhere. }\end{cases}
$$

Since the filters $P_{i}\left(e^{j \omega}\right)$ are nonoverlapping for any two values of $i$, we can define a composite filter

$$
P\left(e^{j \omega}\right)=\sum_{i=0}^{M-1} \hat{\lambda}_{i}\left(e^{j \omega}\right)=\sum_{i=0}^{M-1} \lambda_{i}\left(e^{j \omega M}\right) P_{i}\left(e^{j \omega}\right) / \sqrt{M}
$$




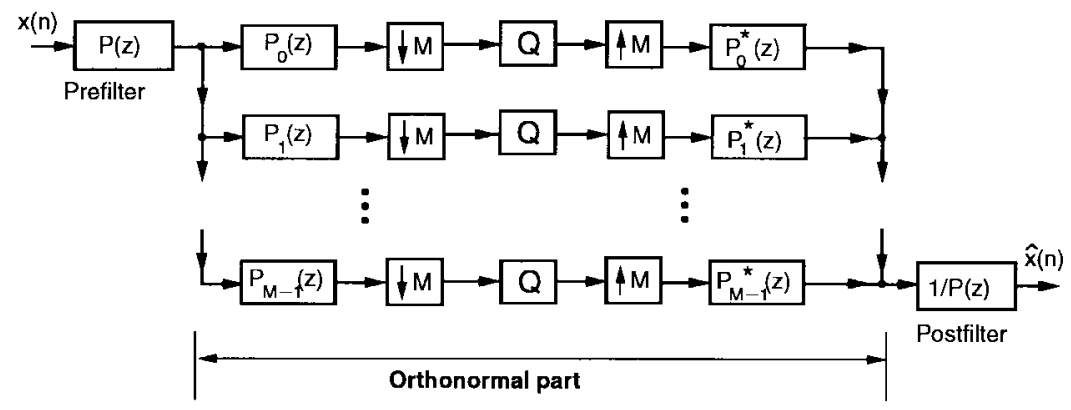

Fig. 11. The prefiltered orthonormal filter bank. Note that $z$ is an abbreviation for $\exp (j \omega)$.

and redraw the system as in Fig. 11. Since $\lambda_{i}\left(e^{j \omega}\right)$ are the half-whitening solutions for the disjoint portions of the input psd created by the analysis system $\left\{P_{i}\left(e^{j \omega}\right)\right\}$, it follows that we can take $P\left(e^{j \omega}\right)$ to be the half-whitening filter for the input $x(n)$, namely $P\left(e^{j \omega}\right)=1 / S_{x x}^{1 / 4}\left(e^{j \omega}\right)$.

\section{Remarks:}

1. The output of $P\left(e^{j \omega}\right)$ has the psd $\sqrt{S_{x x}\left(e^{j \omega}\right)}$. However it can be verified [24] that the optimum orthonormal filter bank for $S_{x x}\left(e^{j \omega}\right)$ is also optimal for $\sqrt{S_{x x}\left(e^{j \omega}\right)}$. So the orthonormal system $\left\{P_{i}\left(e^{j \omega}\right)\right\}$ can be regarded as optimal either for the primary input $x(n)$ or for the output of the prefilter $P\left(e^{j \omega}\right)$.

2. In an earlier work [7], the above combination of $P\left(e^{j \omega}\right)$ and $\left\{P_{i}\left(e^{j \omega}\right)\right\}$ was shown to be optimum among the class of prefiltered orthonormal filter banks (i.e., filter banks restricted to the form of Fig. 11). At the time of this writing we do not know if the most general optimal biorthogonal system can always be represented in this form; but at least for a restricted class of power spectra this can be shown to be the case (Section VII).

3. The optimal prefiltered orthonormal filter bank has the reconstruction error

$$
\begin{aligned}
\mathcal{E} & =c M 2^{-2 b} \phi_{\eta}^{1 / M} \\
& =c M 2^{-2 b}\left(\prod_{i=0}^{M-1} \int_{0}^{2 \pi} \sqrt{\eta_{i}\left(e^{j \omega}\right)} d \omega / 2 \pi\right)^{2 / M}
\end{aligned}
$$

and coding gain

$$
G_{\mathrm{SBC}}(M)=\frac{\sigma_{x}^{2}}{\left(\prod_{i=0}^{M-1} \int_{0}^{2 \pi} \sqrt{\eta_{i}\left(e^{j \omega}\right)} d \omega / 2 \pi\right)^{2 / M}} .
$$

Compare this to $G_{\perp}(M)=\sigma_{x}^{2} /\left(\prod_{i=0}^{M-1} \int_{0}^{2 \pi} \eta_{i}\left(e^{j \omega}\right)\right.$ $d \omega / 2 \pi)^{1 / M}$ which is the optimal orthonormal subband coder gain (10). The improvement therefore is due to the simple fact that $\int_{0}^{2 \pi} \eta_{i}\left(e^{j \omega}\right) d \omega / 2 \pi \geq\left(\int_{0}^{2 \pi}\right.$ $\left.\sqrt{\eta_{i}\left(e^{j \omega}\right)} d \omega / 2 \pi\right)^{2}$, which is a consequence of the Cauchy-Schwartz inequality.

\section{BOUNDS ON THE RECONSTRUCTION ERROR}

At the time of this writing, we do not know if (18) is true for the most general biorthogonal case, but we will derive some other bounds which add insight to the problem. The reconstruction error under optimal bit allocation is given by
$\mathcal{E}=c M 2^{-2 b} \phi^{1 / M}$ (Section II). We can derive useful lower bounds on this by deriving bounds on $\phi$. Let $\mathbf{e}_{i}\left(e^{j \omega}\right)$ and $\mathbf{r}_{i}\left(e^{j \omega}\right)$ denote the columns of $\mathbf{E}^{\dagger}\left(e^{j \omega}\right)$ and $\mathbf{R}\left(e^{j \omega}\right)$, that is,

$$
\begin{aligned}
\mathbf{E}^{\dagger}\left(e^{j \omega}\right) & =\left[\begin{array}{llll}
\mathbf{e}_{0}\left(e^{j \omega}\right) & \mathbf{e}_{1}\left(e^{j \omega}\right) & \cdots & \mathbf{e}_{M-1}\left(e^{j \omega}\right)
\end{array}\right], \\
\mathbf{R}\left(e^{j \omega}\right) & =\mathbf{E}^{-1}\left(e^{j \omega}\right) \\
& =\left[\begin{array}{llll}
\mathbf{r}_{0}\left(e^{j \omega}\right) & \mathbf{r}_{1}\left(e^{j \omega}\right) & \cdots & \mathbf{r}_{M-1}\left(e^{j \omega}\right)
\end{array}\right]
\end{aligned}
$$

and let $\mathbf{S}_{x x}^{1 / 2}\left(e^{j \omega}\right)$ be the Hermitian positive definite square root of the psd $\mathbf{S}_{x x}\left(e^{j \omega}\right)$, that is, $\mathbf{S}_{x x}\left(e^{j \omega}\right)=\mathbf{S}_{x x}^{1 / 2}\left(e^{j \omega}\right) \mathbf{S}_{x x}^{1 / 2}\left(e^{j \omega}\right)$. Using two forms of the Cauchy-Schwartz (CS) inequality we then get (23), shown at the bottom of the next page. The first Cauchy-Schwartz inequality we have used (CS-1) is $\int|f|^{2} \int|g|^{2} \geq\left(\int|f g|\right)^{2}$ and the second one (CS-2) is $\|\mathbf{u}\|^{2}\|\mathbf{v}\|^{2} \geq\left|\mathbf{u}^{\dagger} \mathbf{v}\right|^{2}$. The above shows that, for any choice of the matrix $\mathbf{E}\left(e^{j \omega}\right)$, the quantity $\phi$ is bounded as

$$
\begin{aligned}
\phi & \geq \phi_{\text {bound }} \\
\triangleq & \triangleq \prod_{i=0}^{M-1}\left|\int_{0}^{2 \pi}\left(\mathbf{E}\left(e^{j \omega}\right) \mathbf{S}_{x x}^{1 / 2}\left(e^{j \omega}\right) \mathbf{E}^{-1}\left(e^{j \omega}\right)\right)_{i i} d \omega / 2 \pi\right|^{2} .
\end{aligned}
$$

Notice that $\phi_{\text {bound }}$ still depends on $\mathbf{E}\left(e^{j \omega}\right)$ and is therefore of limited use. The eigenvalues of the matrix $\mathbf{E}\left(e^{j \omega}\right) \mathbf{S}_{x x}^{1 / 2}\left(e^{j \omega}\right) \mathbf{E}^{-1}\left(e^{j \omega}\right)$ are those of $\mathbf{S}_{x x}^{1 / 2}\left(e^{j \omega}\right)$. But $\mathbf{E}\left(e^{j \omega}\right) \mathbf{S}_{x x}^{1 / 2}\left(e^{j \omega}\right) \mathbf{E}^{-1}\left(e^{j \omega}\right)$ is in general not Hermitian, so we cannot apply Corollary 1 to obtain the conclusion that $\phi \geq \phi_{\eta}$ where $\phi_{\eta}$ is defined in (17).

\section{A. Equality Conditions}

It turns out that when the two CS inequalities in (23) are achieved with equality, then Corollary 1 can be successfully applied. But since the equality condition is a side-constraint, we still cannot conclude that $\phi \geq \phi_{\eta}$ for an arbitrary biorthogonal system. We will explore the equality conditions here purely for future reference. In Appendix IV we show that the three inequalities in (23) become equalities if and only if

$$
\mathbf{E}\left(e^{j \omega}\right) \mathbf{S}_{x x}^{1 / 2}\left(e^{j \omega}\right) \mathbf{E}^{\dagger}\left(e^{j \omega}\right)=\mathcal{D}
$$

for some diagonal matrix $\mathcal{D}$ with constant positive diagonal elements $d_{i}$. Notice that $\mathbf{E}\left(e^{j \omega}\right)$ is not in general unitary, so the left hand side above represents a congruence rather than a similarity transformation. The diagonal elements $d_{i}$ are therefore not the eigenvalues of $\mathbf{S}_{x x}^{1 / 2}\left(e^{j \omega}\right)$. Moreover, the diagonalization of $\mathbf{S}_{x x}^{1 / 2}\left(e^{j \omega}\right)$ by congruence does not necessarily mean that $\mathbf{S}_{x x}\left(e^{j \omega}\right)$ itself is diagonalized. Now, since 
$\mathbf{R}\left(e^{j \omega}\right)=\mathbf{E}^{-1}\left(e^{j \omega}\right),(25)$ is equivalent to $\mathbf{E}\left(e^{j \omega}\right) \mathbf{S}_{x x}^{1 / 2}\left(e^{j \omega}\right)=$ $\mathcal{D} \mathbf{R}^{\dagger}\left(e^{j \omega}\right)$ from which we obtain

$$
\begin{gathered}
\mathcal{D}^{-1 / 2} \mathbf{E}\left(e^{j \omega}\right) \mathbf{S}_{x x}^{1 / 2}\left(e^{j \omega}\right) \mathbf{E}^{-1}\left(e^{j \omega}\right) \mathcal{D}^{1 / 2} \\
=\mathcal{D}^{1 / 2} \mathbf{R}^{\dagger}\left(e^{j \omega}\right) \mathbf{R}\left(e^{j \omega}\right) \mathcal{D}^{1 / 2}
\end{gathered}
$$

Thus, when equality is satisfied in (23), we have

$$
\begin{aligned}
\phi= & \prod_{i=0}^{M-1} \mid \int_{0}^{2 \pi}\left(\mathcal{D}^{-1 / 2} \mathbf{E}\left(e^{j \omega}\right) \mathbf{S}_{x x}^{1 / 2}\left(e^{j \omega}\right)\right. \\
& \left.\times \mathbf{E}^{-1}\left(e^{j \omega}\right) \mathcal{D}^{1 / 2}\right)_{i i} d \omega /\left.2 \pi\right|^{2} \text { (since } \mathcal{D} \text { is diagonal) } \\
= & \prod_{i=0}^{M-1}\left|\int_{0}^{2 \pi}\left(\mathcal{D}^{1 / 2} \mathbf{R}^{\dagger}\left(e^{j \omega}\right) \mathbf{R}\left(e^{j \omega}\right) \mathcal{D}^{1 / 2}\right)_{i i} d \omega / 2 \pi\right|^{2}
\end{aligned}
$$

Since the matrix in (26) is a similarity transform of $\mathbf{S}_{x x}^{1 / 2}\left(e^{j \omega}\right)$ (as seen from the left-hand side) its eigenvalues are those of $\mathbf{S}_{x x}^{1 / 2}\left(e^{j \omega}\right)$. Since the matrix is also Hermitian [as seen from the right hand side of (26)], we can therefore apply Corollary 1 to conclude that whenever (24) holds with equality, we have

$$
\begin{aligned}
\phi & =\prod_{i=0}^{M-1}\left|\int_{0}^{2 \pi}\left(\mathbf{E}\left(e^{j \omega}\right) \mathbf{S}_{x x}^{1 / 2}\left(e^{j \omega}\right) \mathbf{E}^{-1}\left(e^{j \omega}\right)\right)_{i i} d \omega / 2 \pi\right|^{2} \\
& \geq\left(\prod_{i=0}^{M-1} \int_{0}^{2 \pi} \sqrt{\eta_{i}\left(e^{j \omega}\right)} d \omega / 2 \pi\right)^{2}
\end{aligned}
$$

We now explain more explicitly why it is a loss of generality to assume that equality conditions are satisfied by the optimal solution. Note that the quantities $\phi$ and $\phi_{\text {bound }}$ [the first and last lines in (23)] depend on $\mathbf{E}\left(e^{j \omega}\right)$, so let us denote them as $\phi(\mathbf{E})$ and $\phi_{\text {bound }}(\mathbf{E})$. We have shown that $\phi(\mathbf{E}) \geq \phi_{\text {bound }}(\mathbf{E})$ for any choice of $\mathbf{E}$. Now consider two choices of $\mathbf{E}$, namely $\mathbf{E}_{1}$ and $\mathbf{E}_{2}$, such that $\mathbf{E}_{1}$ does not satisfy equality constraints but $\mathbf{E}_{2}$ does. That is,

$$
\phi\left(\mathbf{E}_{1}\right)>\phi_{\text {bound }}\left(\mathbf{E}_{1}\right), \quad \phi\left(\mathbf{E}_{2}\right)=\phi_{\text {bound }}\left(\mathbf{E}_{2}\right) .
$$

In general, we cannot argue that this implies $\phi\left(\mathbf{E}_{1}\right) \geq$ $\phi_{\text {bound }}\left(\mathbf{E}_{2}\right)$. That is why forcing equality constraints on $\mathbf{E}$ during optimization could be a loss of generality. However, in Appendix IV we show that there is no loss of generality in forcing the first inequality in (23) to be an equality. The optimal $\phi$ can therefore be assumed to be of the form of (29), as shown at the bottom of the next page.

\section{B. The Symmetry Property}

From the basic expression (2) for $\phi$, we notice a certain symmetry between the two integrals, since $\mathbf{R}\left(e^{j \omega}\right)$ is the inverse of $\mathbf{E}\left(e^{j \omega}\right)$. More specifically, we will find that if we replace $\mathbf{E}\left(e^{j \omega}\right)$ with $\mathbf{E}^{-\dagger}\left(e^{j \omega}\right) \mathbf{S}_{x x}^{-1 / 2}\left(e^{j \omega}\right)$ (and change $\mathbf{R}\left(e^{j \omega}\right)$ correspondingly) then $\phi$ will not change.

In particular, if the optimal $\mathbf{E}\left(e^{j \omega}\right)$ can be assumed to be unique $^{3}$ (up to a constant diagonal premultiplier matrix $\mathcal{D}$ which does not matter) then $\mathbf{E}\left(e^{j \omega}\right)=$ $\mathcal{D} \mathbf{E}^{-\dagger}\left(e^{j \omega}\right) \mathbf{S}_{x x}^{-1 / 2}\left(e^{j \omega}\right)$, i.e., $\mathbf{E}\left(e^{j \omega}\right) \mathbf{S}_{x x}^{1 / 2}\left(e^{j \omega}\right) \mathbf{E}^{\dagger}\left(e^{j \omega}\right)=\mathcal{D}$. This is precisely the equality condition (25). Thus, in those cases where the optimal $\mathbf{E}\left(e^{j \omega}\right)$ is unique, we can indeed justify that the equality condition (25) is necessary. In this case, the best biorthogonal system satisfies $\phi=\phi_{\eta}$, and it can be implemented as the prefiltered orthonormal filter bank Fig. 11.

\section{INSIGHTFUL SPECIAL CASES}

According to the singular value decomposition (SVD) theorem [9], the nonsingular matrix $\mathbf{E}\left(e^{j \omega}\right)$ can be expressed in

${ }^{3}$ Such uniqueness is not very uncommon; for example, at the end of Section III, we explained situations where the orthonormal filter bank is unique.

$$
\begin{aligned}
\phi= & \prod_{i=0}^{M-1} \int_{0}^{2 \pi}\left(\mathbf{E}\left(e^{j \omega}\right) \mathbf{S}_{x x}\left(e^{j \omega}\right) \mathbf{E}^{\dagger}\left(e^{j \omega}\right)\right)_{i i} d \omega / 2 \pi \\
& \times \int_{0}^{2 \pi}\left(\mathbf{R}^{\dagger}\left(e^{j \omega}\right) \mathbf{R}\left(e^{j \omega}\right)\right)_{i i} d \omega / 2 \pi \\
\geq & \prod_{i=0}^{M-1}\left(\int_{0}^{2 \pi} \sqrt{\left(\mathbf{E}\left(e^{j \omega}\right) \mathbf{S}_{x x}\left(e^{j \omega}\right) \mathbf{E}^{\dagger}\left(e^{j \omega}\right)\right)_{i i}\left(\mathbf{R}^{\dagger}\left(e^{j \omega}\right) \mathbf{R}\left(e^{j \omega}\right)\right)_{i i}} d \omega / 2 \pi\right)^{2} \\
= & \prod_{i=0}^{M-1}\left(\int_{0}^{2 \pi} \sqrt{\left\|\mathbf{S}_{x x}^{1 / 2}\left(e^{j \omega}\right) \mathbf{e}_{i}\left(e^{j \omega}\right)\right\|^{2}\left\|\mathbf{r}_{i}\left(e^{j \omega}\right)\right\|^{2}} d \omega / 2 \pi\right)^{2} \\
\geq & \prod_{i=0}^{M-1}\left(\int_{0}^{2 \pi}\left|\mathbf{e}_{i}^{\dagger}\left(e^{j \omega}\right) \mathbf{S}_{x x}^{1 / 2}\left(e^{j \omega}\right) \mathbf{r}_{i}\left(e^{j \omega}\right)\right| d \omega / 2 \pi\right)^{2}(\mathrm{CS}-2) \\
\geq & \prod_{i=0}^{M-1}\left|\int_{0}^{2 \pi} \mathbf{e}_{i}^{\dagger}\left(e^{j \omega}\right) \mathbf{S}_{x x}^{1 / 2}\left(e^{j \omega}\right) \mathbf{r}_{i}\left(e^{j \omega}\right) d \omega / 2 \pi\right|^{2} \\
= & \prod_{i=0}^{M-1}\left|\int_{0}^{2 \pi}\left(\mathbf{E}\left(e^{j \omega}\right) \mathbf{S}_{x x}^{1 / 2}\left(e^{j \omega}\right) \mathbf{E}^{-1}\left(e^{j \omega}\right)\right)_{i i} d \omega / 2 \pi\right|^{2}
\end{aligned}
$$




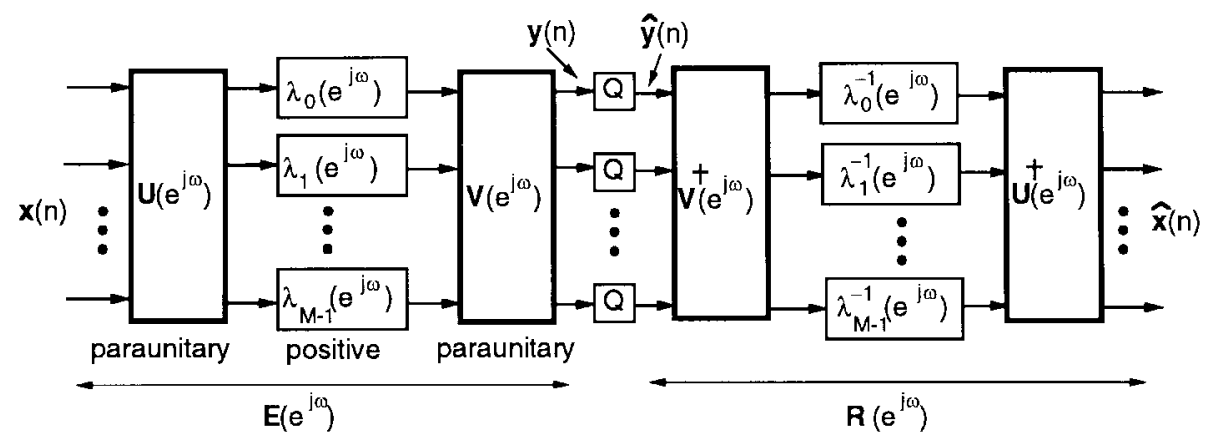

Fig. 12. The general form of a biorthogonal subband coder.

the form

$$
\mathbf{E}\left(e^{j \omega}\right)=\mathbf{V}\left(e^{j \omega}\right) \boldsymbol{\Lambda}\left(e^{j \omega}\right) \mathbf{U}\left(e^{j \omega}\right)
$$

where $\mathbf{U}\left(e^{j \omega}\right)$ and $\mathbf{V}\left(e^{j \omega}\right)$ are unitary and $\boldsymbol{\Lambda}\left(e^{j \omega}\right)$ is a diagonal matrix with positive diagonal elements $\lambda_{i}\left(e^{j \omega}\right)$. So the most general biorthogonal filter bank can be drawn as in Fig. 12. We can express the objective function $\phi$ in terms of the SVD parameters by substituting (30) into (2). Using the unitarity of $\mathbf{U}\left(e^{j \omega}\right)$ and $\mathbf{V}\left(e^{j \omega}\right)$ this simplifies to

$$
\begin{aligned}
\phi= & \prod_{i=0}^{M-1} \int_{0}^{2 \pi}\left(\mathbf{V} \mathbf{\Lambda} \mathbf{U} \mathbf{S}_{x x} \mathbf{U}^{\dagger} \boldsymbol{\Lambda} \mathbf{V}^{\dagger}\right)_{i i} d \omega / 2 \pi \\
& \times \prod_{i=0}^{M-1} \int_{0}^{2 \pi}\left(\mathbf{V} \boldsymbol{\Lambda}^{-2} \mathbf{V}^{\dagger}\right)_{i i} d \omega / 2 \pi
\end{aligned}
$$

where the argument $\left(e^{j \omega}\right)$ is deleted for simplicity. Similarly the bound (24) can be expressed as

$$
\phi \geq \prod_{i=0}^{M-1}\left|\int_{0}^{2 \pi}\left(\mathbf{V} \boldsymbol{\Lambda} \mathbf{U S}_{x x}^{1 / 2} \mathbf{U}^{\dagger} \boldsymbol{\Lambda}^{-1} \mathbf{V}^{\dagger}\right)_{i i} d \omega / 2 \pi\right|^{2}
$$

Observation 1: Consider the special case where $\mathbf{V}\left(e^{j \omega}\right)=$ I. What is the smallest value of $\phi$ that can be achieved by this system? From (32) we see that, in this case

$$
\begin{aligned}
\phi & \geq \prod_{i=0}^{M-1}\left|\int_{0}^{2 \pi}\left(\mathbf{\Lambda} \mathbf{U} \mathbf{S}_{x x}^{1 / 2} \mathbf{U}^{\dagger} \boldsymbol{\Lambda}^{-1}\right)_{i i} d \omega / 2 \pi\right|^{2} \\
& =\prod_{i=0}^{M-1}\left|\int_{0}^{2 \pi}\left(\mathbf{U S}_{x x}^{1 / 2} \mathbf{U}^{\dagger}\right)_{i i} d \omega / 2 \pi\right|^{2}
\end{aligned}
$$

since $\boldsymbol{\Lambda}\left(e^{j \omega}\right)$ is diagonal. The square root $\mathbf{S}_{x x}^{1 / 2}\left(e^{j \omega}\right)$ is Hermitian, so the product $\mathbf{U}\left(e^{j \omega}\right) \mathbf{S}_{x x}^{1 / 2}\left(e^{j \omega}\right) \mathbf{U}^{\dagger}\left(e^{j \omega}\right)$ is also Hermitian, and we can use Corollary 1 to further simplify this bound into a useful form. Thus, since $\sqrt{\eta_{i}\left(e^{j \omega}\right)}$ is the majorized set of eigenvalues of $\mathbf{S}_{x x}^{1 / 2}\left(e^{j \omega}\right)$, we indeed have

$$
\phi \geq\left(\prod_{i=0}^{M-1} \int_{0}^{2 \pi} \sqrt{\eta_{i}\left(e^{j \omega}\right)} d \omega / 2 \pi\right)^{2}=\phi_{\eta}
$$

Observation 2: Now assume that in the system of Fig. 12, the paraunitary matrix $\mathbf{U}\left(e^{j \omega}\right)$ is chosen to perform total decorrelation. Then what is a lower bound on $\phi$ obtainable by optimizing $\boldsymbol{\Lambda}\left(e^{j \omega}\right)$ and $\mathbf{V}\left(e^{j \omega}\right)$ ? In this case, we have $\mathbf{U}\left(e^{j \omega}\right) \mathbf{S}_{x x}\left(e^{j \omega}\right) \mathbf{U}^{\dagger}\left(e^{j \omega}\right)=\mathcal{S}\left(e^{j \omega}\right)$ where $\mathcal{S}\left(e^{j \omega}\right)$ is diagonal. Since $\mathbf{S}_{x x}^{1 / 2}\left(e^{j \omega}\right)$ is the Hermitian square root of $\mathbf{S}_{x x}\left(e^{j \omega}\right)$, we have

$$
\mathbf{S}_{x x}^{1 / 2}\left(e^{j \omega}\right)=\mathbf{U}^{\dagger}\left(e^{j \omega}\right) \mathcal{S}^{1 / 2}\left(e^{j \omega}\right) \mathbf{U}\left(e^{j \omega}\right)
$$

so that $\mathbf{U}\left(e^{j \omega}\right) \mathbf{S}_{x x}^{1 / 2}\left(e^{j \omega}\right) \mathbf{U}^{\dagger}\left(e^{j \omega}\right)$ is the diagonal matrix $\mathcal{S}^{1 / 2}$. Thus, (32) yields

$$
\begin{aligned}
\phi & \geq \prod_{i=0}^{M-1}\left|\int_{0}^{2 \pi}\left(\mathbf{V} \boldsymbol{\Lambda} \mathcal{S}^{1 / 2} \boldsymbol{\Lambda}^{-1} \mathbf{V}^{\dagger}\right)_{i i} d \omega / 2 \pi\right|^{2} \\
& =\prod_{i=0}^{M-1}\left|\int_{0}^{2 \pi}\left(\mathbf{V} \mathcal{S}^{1 / 2} \mathbf{V}^{\dagger}\right)_{i i} d \omega / 2 \pi\right|^{2}
\end{aligned}
$$

since $\mathcal{S}^{1 / 2}$ is diagonal. The matrix $\mathbf{V} \mathcal{S}^{1 / 2} \mathbf{V}^{\dagger}$ is Hermitian, and we can again apply Corollary 1 to obtain

$$
\phi \geq\left(\prod_{i=0}^{M-1} \int_{0}^{2 \pi} \sqrt{\eta_{i}\left(e^{j \omega}\right)} d \omega / 2 \pi\right)^{2}=\phi_{\eta} .
$$

Thus, if $\mathbf{U}\left(e^{j \omega}\right)$ is a decorrelating matrix, then $\phi \geq \phi_{\eta}$. Summarizing these observations, we have shown the following.

1) If we impose the condition $\mathbf{V}\left(e^{j \omega}\right)=\mathbf{I}$ and then optimize $\mathbf{U}\left(e^{j \omega}\right)$ and $\boldsymbol{\Lambda}\left(e^{j \omega}\right)$, then the resulting $\phi$ satisfies $\phi \geq \phi_{\eta}$.

2) Instead of imposing the condition $\mathbf{V}\left(e^{j \omega}\right)=\mathbf{I}$, suppose we impose the condition that $\mathbf{U}\left(e^{j \omega}\right)$ be chosen to perform total decorrelation of its output. If we now try to optimize $\boldsymbol{\Lambda}\left(e^{j \omega}\right)$ and $\mathbf{V}\left(e^{j \omega}\right)$, then the resulting $\phi$ again satisfies $\phi \geq \phi_{\eta}$.

3) In any case, recall that the solution $\phi=\phi_{\eta}$ can be achieved by the following choice of matrices: set $\mathbf{V}=$ $\mathbf{I}$, take $\mathbf{U}\left(e^{j \omega}\right)$ to be the optimal paraunitary matrix (the one that performs total decorrelation and spectral majorization), and take the diagonal elements of $\boldsymbol{\Lambda}\left(e^{j \omega}\right)$

$$
\phi=\left(\prod_{i=0}^{M-1} \int_{0}^{2 \pi} \sqrt{\left(\mathbf{E}\left(e^{j \omega}\right) \mathbf{S}_{x x}\left(e^{j \omega}\right) \mathbf{E}^{\dagger}\left(e^{j \omega}\right)\right)_{i i}\left(\mathbf{R}^{\dagger}\left(e^{j \omega}\right) \mathbf{R}\left(e^{j \omega}\right)\right)_{i i}} d \omega / 2 \pi\right)^{2}
$$


to be the half-whitening filters for the outputs of $\mathbf{U}\left(e^{j \omega}\right)$ (as in Fig. 8(c) earlier).

Purpose of $U$ : Notice, however, that it has not been shown here or in the literature (though it is most likely true) that $\mathbf{U}\left(e^{j \omega}\right)$ can be chosen to be a decorrelating matrix without loss of generality of the biorthogonal subband coder. So it is not clear, at the time of this writing, whether the more general biorthogonal system with $\mathbf{V}\left(e^{j \omega}\right) \neq \mathbf{I}$ still satisfies $\phi \geq \phi_{\eta}$. If it could do better than this, then the optimal biorthogonal system will be such that the output of $\mathbf{U}\left(e^{j \omega}\right)$ is not totally decorrelated (otherwise observation 2 is violated). Then what is the role of $\mathbf{U}\left(e^{j \omega}\right)$ in the optimal system? Return to the general expression for $\phi$ in (31). The optimal choice of the triplet $\left\{\mathbf{U}\left(e^{j \omega}\right), \boldsymbol{\Lambda}\left(e^{j \omega}\right), \mathbf{V}\left(e^{j \omega}\right)\right\}$ is such that if we fix $\boldsymbol{\Lambda}\left(e^{j \omega}\right)$ and $\mathbf{V}\left(e^{j \omega}\right)$ and perturb $\mathbf{U}\left(e^{j \omega}\right)$ from the optimal value, then the product of the subband variances (i.e., the product of $\left.\int_{0}^{2 \pi}\left(\mathbf{V} \boldsymbol{\Lambda} \mathbf{U} \mathbf{S}_{x x} \mathbf{U}^{\dagger} \boldsymbol{\Lambda} \mathbf{V}^{\dagger}\right)_{i i} d \omega / 2 \pi\right)$ can only increase. In this limited sense $\mathbf{U}\left(e^{j \omega}\right)$ serves to minimize the product of subband variances. This also gives rise to the idea of a family of power spectra.

Theorem 2-Power Spectrum Family: The minimum value of the quantity $\phi$ (hence, the reconstruction error $\mathcal{E}$ ) is the same for the entire family of input psd matrices with the same set of majorized eigenvalues $\left\{\eta_{i}\left(e^{j \omega}\right)\right\}$. Moreover the same $\boldsymbol{\Lambda}\left(e^{j \omega}\right)$ and $\mathbf{V}\left(e^{j \omega}\right)$ can be used to achieve the minimum $\phi$ for all members of the family; only the choice of $\mathbf{U}\left(e^{j \omega}\right)$ depends on the member.

Thus, the quantity $\mathbf{U}\left(e^{j \omega}\right) \mathbf{S}_{x x}\left(e^{j \omega}\right) \mathbf{U}^{\dagger}\left(e^{j \omega}\right)$, which is the psd presented to the diagonal matrix $\boldsymbol{\Lambda}\left(e^{j \omega}\right)$, is the same for this family. The role of $\mathbf{U}\left(e^{j \omega}\right)$ is to turn any member $\mathbf{S}_{x x}\left(e^{j \omega}\right)$ from this family into a "standard psd of the family" to be fed into $\boldsymbol{\Lambda}\left(e^{j \omega}\right)$.

Proof: Let $\mathbf{S}_{i}\left(e^{j \omega}\right), i=1,2$ be two psd matrices in this family, with minimum values $\phi=\phi_{i}$ achieved by the combination $\left\{\mathbf{U}_{i}\left(e^{j \omega}\right), \boldsymbol{\Lambda}_{i}\left(e^{j \omega}\right), \mathbf{V}_{i}\left(e^{j \omega}\right)\right\}$ Thus,

$$
\begin{aligned}
\phi_{1}= & \prod_{i=0}^{M-1} \int_{0}^{2 \pi}\left(\mathbf{V}_{1} \boldsymbol{\Lambda}_{1} \mathbf{U}_{1} \mathbf{S}_{1} \mathbf{U}_{1}^{\dagger} \boldsymbol{\Lambda}_{1} \mathbf{V}_{1}^{\dagger}\right)_{i i} d \omega / 2 \pi \\
& \times \prod_{i=0}^{M-1} \int_{0}^{2 \pi}\left(\mathbf{V}_{1} \boldsymbol{\Lambda}_{1}^{-2} \mathbf{V}_{1}^{\dagger}\right)_{i i} d \omega / 2 \pi \\
\phi_{2}= & \prod_{i=0}^{M-1} \int_{0}^{2 \pi}\left(\mathbf{V}_{2} \boldsymbol{\Lambda}_{2} \mathbf{U}_{2} \mathbf{S}_{2} \mathbf{U}_{2}^{\dagger} \boldsymbol{\Lambda}_{2} \mathbf{V}_{2}^{\dagger}\right)_{i i} d \omega / 2 \pi \\
& \times \prod_{i=0}^{M-1} \int_{0}^{2 \pi}\left(\mathbf{V}_{2} \boldsymbol{\Lambda}_{2}^{-2} \mathbf{V}_{2}^{\dagger}\right)_{i i} d \omega / 2 \pi .
\end{aligned}
$$

We assume $\phi_{1}<\phi_{2}$ and obtain a conflict. Note that there is a unitary $\mathbf{U}_{3}\left(e^{j \omega}\right)$ such that $\mathbf{U}_{1} \mathbf{S}_{1} \mathbf{U}_{1}^{\dagger}=\mathbf{U}_{3} \mathbf{S}_{2} \mathbf{U}_{3}^{\dagger}$ [because $\mathbf{S}_{1}\left(e^{j \omega}\right)$ and $\mathbf{S}_{2}\left(e^{j \omega}\right)$ are related as $\mathbf{S}_{1}\left(e^{j \omega}\right)=$ $\mathbf{A}\left(e^{j \omega}\right) \mathbf{S}_{2}\left(e^{j \omega}\right) \mathbf{A}^{\dagger}\left(e^{j \omega}\right)$ for some unitary $\left.\mathbf{A}\left(e^{j \omega}\right)\right]$. Thus, for the psd $\mathbf{S}_{2}\left(e^{j \omega}\right)$, we can now achieve a smaller $\phi$, namely $\phi=\phi_{1}<\phi_{2}$, by choosing $\mathbf{U}\left(e^{j \omega}\right)=\mathbf{U}_{3}\left(e^{j \omega}\right)$, $\boldsymbol{\Lambda}\left(e^{j \omega}\right)=\boldsymbol{\Lambda}_{1}\left(e^{j \omega}\right)$, and $\mathbf{V}\left(e^{j \omega}\right)=\mathbf{V}_{1}\left(e^{j \omega}\right)$, contradicting minimality of $\phi_{2}$.

\section{BOUNDS BASED ON DETERMINANTS}

We can obtain another bound for $\phi$ in terms of the determinant of the psd matrix $\mathbf{S}_{x x}\left(e^{j \omega}\right)$, namely,

$$
\phi \geq\left(\int_{0}^{2 \pi}\left(\operatorname{det} \mathbf{S}_{x x}\right)^{1 / 2 M} d \omega / 2 \pi\right)^{2 M} .
$$

This can be shown based on standard inequalities used earlier in the paper, and an additional tool, namely Minkowski's inequality for sums of determinants [9, p. 482]. Actually we shall need the integral version of this which can be found in [27]. This says that if $\mathbf{F}\left(e^{j \omega}\right)$ is positive definite for all $\omega$, then

$$
\begin{aligned}
& \operatorname{det} \int_{0}^{2 \pi} \mathbf{F}\left(e^{j \omega}\right) d \omega / 2 \pi \\
& \quad \geq\left(\int_{0}^{2 \pi}\left(\operatorname{det} \mathbf{F}\left(e^{j \omega}\right)\right)^{1 / M} d \omega / 2 \pi\right)^{M}
\end{aligned}
$$

where the size of $\mathbf{F}\left(e^{j \omega}\right)$ is $M \times M$. Here is how we prove (37):

$$
\begin{aligned}
\phi= & \prod_{i=0}^{M-1} \int_{0}^{2 \pi}\left(\mathbf{E}\left(e^{j \omega}\right) \mathbf{S}_{x x}\left(e^{j \omega}\right) \mathbf{E}^{\dagger}\left(e^{j \omega}\right)\right)_{i i} d \omega / 2 \pi \\
& \times \prod_{i=0}^{M-1} \int_{0}^{2 \pi}\left(\mathbf{R}^{\dagger}\left(e^{j \omega}\right) \mathbf{R}\left(e^{j \omega}\right)\right)_{i i} d \omega / 2 \pi \\
\geq & \operatorname{det}\left(\int_{0}^{2 \pi} \mathbf{E}\left(e^{j \omega}\right) \mathbf{S}_{x x}\left(e^{j \omega}\right) \mathbf{E}^{\dagger}\left(e^{j \omega}\right) d \omega / 2 \pi\right) \\
& \times \operatorname{det}\left(\int_{0}^{2 \pi} \mathbf{R}^{\dagger}\left(e^{j \omega}\right) \mathbf{R}\left(e^{j \omega}\right) d \omega / 2 \pi\right) \\
\geq & \left(\int_{0}^{2 \pi}\left(\operatorname{det}\left(\mathbf{E}\left(e^{j \omega}\right) \mathbf{S}_{x x}\left(e^{j \omega}\right) \mathbf{E}^{\dagger}\left(e^{j \omega}\right)\right)^{1 / M} d \omega / 2 \pi\right)^{M}\right. \\
& \left.\times \int_{0}^{2 \pi}\left(\operatorname{det}\left(\mathbf{R}^{\dagger}\left(e^{j \omega}\right) \mathbf{R}\left(e^{j \omega}\right)\right)\right)^{1 / M} d \omega / 2 \pi\right)^{M} \\
\geq & \left(\int _ { 0 } ^ { 2 \pi } \left(\operatorname{det}\left(\mathbf{E}\left(e^{j \omega}\right) \mathbf{S}_{x x}\left(e^{j \omega}\right) \mathbf{E}^{\dagger}\left(e^{j \omega}\right)\right)\right.\right. \\
& \left.\left.\times \operatorname{det}\left(\mathbf{R}^{\dagger}\left(e^{j \omega}\right) \mathbf{R}\left(e^{j \omega}\right)\right)\right)^{1 / 2 M} d \omega / 2 \pi\right)^{2 M} \\
= & \left(\int_{0}^{2 \pi}\left(\operatorname{det} \mathbf{S}_{x x}\right)^{1 / 2 M} d \omega / 2 \pi\right)^{2 M} \cdot
\end{aligned}
$$

The first inequality is Hadamard's inequality for positive definite matrices, the second one is Minkowski's inequality for determinants, and the third inequality is Cauchy-Schwartz inequality for integrals. Thus the bound has been established. Notice that for the orthonormal case $\mathbf{R}^{\dagger}\left(e^{j \omega}\right) \mathbf{R}\left(e^{j \omega}\right)=\mathbf{I}$, and from the third line above we get the tighter bound

$$
\phi \geq\left(\int_{0}^{2 \pi}\left(\operatorname{det} \mathbf{S}_{x x}\right)^{1 / M} d \omega / 2 \pi\right)^{M} \quad \text { (orthonormal case). }
$$

Whether these bounds are useful or not depends on the case under consideration. For example, imagine that $\mathbf{S}_{x x}\left(e^{j \omega}\right)$ is diagonal such that at least one diagonal element is zero for each $\omega$. Then $\operatorname{det} \mathbf{S}_{x x}=0$ for all $\omega$, and the above bound says nothing more than the obvious fact that $\phi \geq 0$. However, there are some useful applications of this bound. For example 
we will exhibit a class of power spectra for which the special form of Fig. 8(c) is indeed the optimal biorthogonal system.

\section{A. Inputs for Which Prefiltered Orthonormal System Is Best}

Suppose the psd matrix $\mathbf{S}_{x x}\left(e^{j \omega}\right)$ has majorized eigenvalues of the form

$$
\eta_{i}\left(e^{j \omega}\right)=c_{i} \eta\left(e^{j \omega}\right)
$$

where $c_{i}$ are constants. That is, all eigenvalues are scaled versions of the same basic shape $\eta\left(e^{j \omega}\right)$. Thus, $\operatorname{det} \mathbf{S}_{x x}\left(e^{j \omega}\right)=$ $\left(\eta\left(e^{j \omega}\right)\right)^{M} \prod_{i} c_{i}$ and we verify by direct computation that (37) implies

$$
\begin{aligned}
\phi & \geq\left(\int_{0}^{2 \pi} \sqrt{\eta\left(e^{j \omega}\right)} d \omega / 2 \pi\right)^{2 M} \times \prod_{i=0}^{M-1} c_{i} \\
& =\left(\prod_{i=0}^{M-1} \int_{0}^{2 \pi} \sqrt{\eta_{i}\left(e^{j \omega}\right)} d \omega / 2 \pi\right)^{2}=\phi_{\eta} .
\end{aligned}
$$

This shows that the special biorthogonal system of Fig. 8(c) is indeed the optimal biorthogonal system for the psd $\mathbf{S}_{x x}\left(e^{j \omega}\right)$. The output of the optimal orthonormal part $\mathbf{U}\left(e^{j \omega}\right)$ has the psd matrix $\mathbf{S}_{w w}\left(e^{j \omega}\right)=\eta\left(e^{j \omega}\right) \mathbf{C}$ where $\mathbf{C}$ is diagonal with constant diagonal elements $c_{i}$. We can therefore take the halfwhiteners in Fig. 8(c) to be $\lambda_{i}\left(e^{j \omega}\right)=1 /\left[\eta\left(e^{j \omega}\right)\right]^{1 / 4}$ for all $i$. Two further special cases are interesting:

1. Special Case 1: Suppose $\eta\left(e^{j \omega}\right)$ is a constant, i.e., the majorized eigenvalues $\eta_{i}\left(e^{j \omega}\right)=c_{i}$ for all $i$. Then the half-whitening part is unnecessary. In this case, the optimal orthonormal subband coder is also the optimal biorthogonal subband coder!

2. Special Case 2-Constant Diagonal psd: Suppose $\mathbf{S}_{x x}$ $\left(e^{j \omega}\right)$ itself is diagonal with constant diagonal elements. Then (37) reduces to $\phi \geq \prod_{i=0}^{M-1} \sigma_{x_{i}}^{2}$, and this bound can be "achieved" trivially by choosing $\mathbf{E}\left(e^{j \omega}\right)=$ $\mathbf{R}\left(e^{j \omega}\right)=\mathbf{I}$ (and performing optimal bit allocation, of course). As one might have intuitively guessed, no filters are necessary; decorrelation, majorization, and half-whitening are already implicit.

The significance of these observations is deeper than it appears at first sight. To appreciate this, consider again the system of Fig. 1, and assume that the scalar input $x(n)$ has psd of the form demonstrated in Fig. 13(a). That is, if we partition the frequency domain $[0,2 \pi)$ into $M$ uniform regions, the psd has the same shape in each region except for a scale factor $c_{i}$. Mathematically,

$$
S_{x x}\left(e^{j \omega}\right)=\sum_{i=0}^{M-1} c_{i} g\left(e^{j(\omega-2 \pi i / M)}\right)
$$

in region $i$, where $g\left(e^{j \omega}\right)$ is as shown in Fig. 13(b). If we choose the analysis filter responses as in Fig. 13(c), then we have decorrelation as well as spectral majorization (possibly subject to renumbering of filters). The decimated subband psd are given by $\eta_{i}\left(e^{j \omega}\right)=c_{i} g\left(e^{j \omega / M}\right)$ for $(0,2 \pi]$ (and repeat with period $2 \pi)$. This shows that the majorized eigenvalues indeed have the form (40). The optimal biorthogonal filter bank in

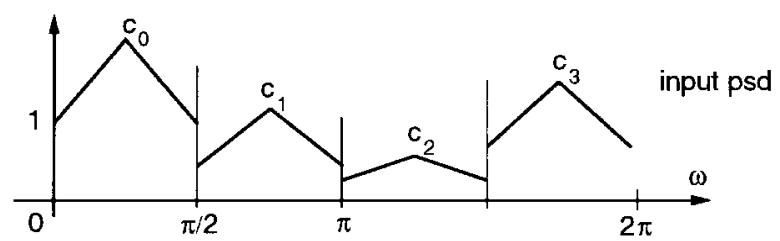

(a)

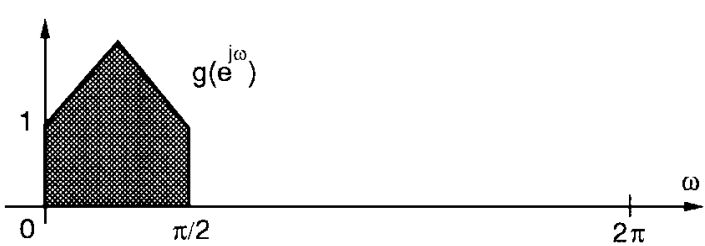

(b)

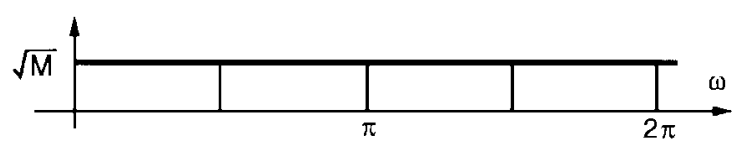

(c)

Fig. 13. (a) A special kind of input psd, (b) the basic shape generating the psd, and (c) ideal brickwall analysis filters.

polyphase form is therefore as in Fig. 8(c). In scalar form, the optimum system is therefore the prefiltered orthonormal system of Fig. 11.

This result can readily be extended to more general psd. For this, notice that the partitioning of Fig. 13(a) is a special case of aliasfree $(M)$ tiling or partitioning. Such a partition is one in which the region $[0,2 \pi)$ is divided into $M$ regions $\mathcal{R}_{i}$ such that each region has total width $2 \pi / M$, and furthermore, if $\mathcal{R}_{i}$ is shifted by $2 \pi k / M$ for $0<k<M$, the result does not overlap with $\mathcal{R}_{i}$. The optimal orthonormal example of Fig. 6(b) shows an example of such a tiling; the region $\mathcal{R}_{i}$, which is the passband of the $i$ th filter, may not be in one piece, though its total width is $2 \pi / M$. Given any psd, there always exists an optimal orthonormal subband coder which performs aliasfree $(M)$ tiling [24].

Now suppose $S_{x x}\left(e^{j \omega}\right)$ has the property that there exists an alias free tiling $\left\{\mathcal{R}_{i}\right\}$ such that $S_{x x}\left(e^{j \omega}\right)$ has the "same basic shape" in each region. More precisely, this means that if we use an ideal filter with passband region $\mathcal{R}_{i}$ and decimate the output by $M$, then the result is $c_{i} \eta\left(e^{j \omega}\right)$ where $c_{i}$ are constants. For such a psd, we can verify using the preceding arguments that Fig. 11 is still the best biorthogonal filter bank. We summarize these discussions as follows (review Section IV-B here).

Theorem 3-Sufficiency of Prefiltered Orthonormal Filter Bank: In the subband coder of Fig. 1(a), suppose the input psd $S_{x x}\left(e^{j \omega}\right)$ has the same basic shape (in the sense explained above) in each region $\mathcal{R}_{i}$ of some aliasfree $(M)$ tiling of $[0,2 \pi)$. Then the optimum biorthogonal solution can be represented as the prefiltered orthonormal filter bank of Fig. 11, where $P(z)$ is the half-whitening filter for the input (i.e., $\left.P\left(e^{j \omega}\right)=1 /\left[S_{x x}\left(e^{j \omega}\right)\right]^{1 / 4}\right)$ and $\left\{P_{k}\left(e^{j \omega}\right)\right\}$ is the optimum orthonormal analysis bank for $x(n)$.

As mentioned in Section IV-B, $\left\{P_{k}\left(e^{j \omega}\right)\right\}$ can also be regarded as optimal for the output of $P(z)$. In fact, the prefilter 


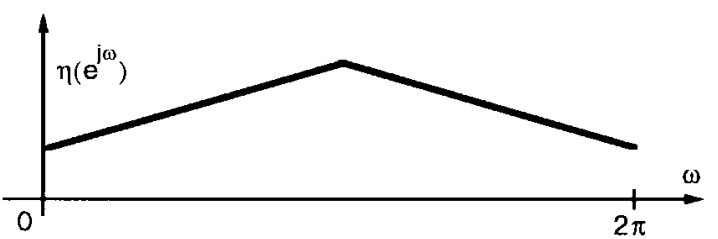

(a)

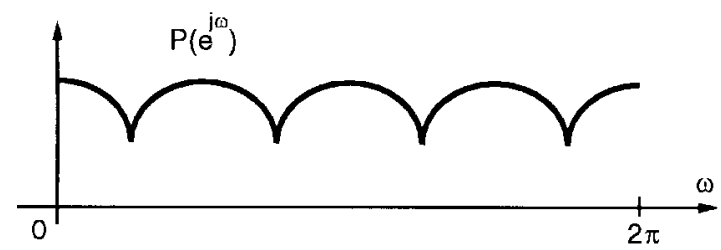

(b)

Fig. 14. (a) The majorized eigenvalue determining the prefilter and (b) the optimal prefilter.

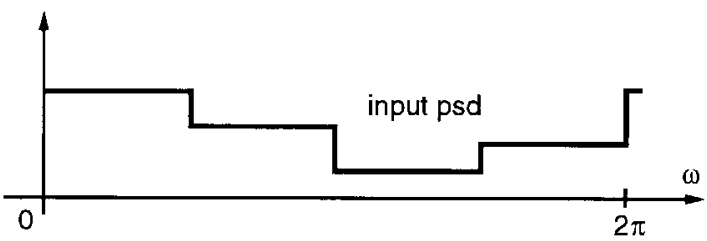

Fig. 15. A piecewise constant psd for which orthonormal SBC is as good as biorthogonal.

can further be simplified. Recall its construction described in (19) and (20). Since constant scale factors in front of $\lambda_{i}\left(e^{j \omega}\right)$ do not affect reconstruction errors, we can let $\lambda_{i}\left(e^{j \omega}\right)=$ $\left[\eta\left(e^{j \omega}\right)\right]^{-1 / 4}$ for all $i$. Then from (20)

$$
\begin{aligned}
P\left(e^{j \omega}\right) & =\left(\eta\left(e^{j \omega M}\right)\right)^{-1 / 4} \sum_{i=0}^{M-1} P_{i}\left(e^{j \omega}\right) / \sqrt{M} \\
& =\left(\eta\left(e^{j \omega M}\right)\right)^{-1 / 4}
\end{aligned}
$$

since $\left\{P_{i}\left(e^{j \omega}\right)\right\}$ are nonoverlapping filters tiling $[0,2 \pi)$, each having a passband response $\sqrt{M}$. Fig. 14 demonstrates this for the example of Fig. 13.

A special case of the previous theorem arises when the psd $S_{x x}\left(e^{j \omega}\right)$ is a constant $c_{i}$ in each alias-free $(M)$ region $\mathcal{R}_{i}$. In this case, we have $\eta\left(e^{j \omega}\right)=1$ and the prefilter $P\left(e^{j \omega}\right)=1$. That is, the optimal biorthogonal solution is in fact orthonormal! Such a psd is demonstrated in Fig. 15, and is a piecewise constant. We summarize this observation as a corollary, which was also proved in [24] differently.

Corollary 2-Sufficiency of Orthonormal Filter Bank: With reference to the subband coder of Fig. 1(a), the following statements are equivalent:

1. the input psd $S_{x x}\left(e^{j \omega}\right)$ is a constant in each region $\mathcal{R}_{i}$ of some aliasfree $(M)$ tiling of $[0,2 \pi)$;

2. the optimum orthonormal solution is also the optimum biorthogonal solution.

Proof: We already showed that the first statement implies the second. Now consider Fig. 8(c) where the optimal orthonormal part is assumed to come from an ideal aliasfree $(M)$ partition. If statement 2 is true, the diagonal psd matrix $\mathbf{S}_{w w}\left(e^{j \omega}\right)$ has to have constant elements $c_{i}$ (otherwise, the half-whiteners $\lambda_{i}\left(e^{j \omega}\right)$ could decrease the reconstruction error). This implies that the original psd has constant value $c_{i}$ in each alias free region $\mathcal{R}_{i}$. This shows that statement 2 implies statement 1 .

\section{CONCLUDING REMARKS}

All results in this paper are for the biorthogonal subband coder of Fig. 1(a) which has the polyphase representation shown in Fig. 1(b). All discussions are for uniform filter banks (identical decimation in all subbands) with unrestricted filter orders. We assume also that the subband quantizers perform optimum bit allocation at a fixed average rate $b$. The expression for the reconstruction error under optimal bit allocation is given by (1) where $\phi$ is defined in (2). Optimization of the filters in the subband coder is therefore equivalent to minimizing $\phi$. For the orthonormal case (Section III), the necessary and sufficient condition for optimality is that the decimated subband signals $y_{i}(n)$ be totally decorrelated and spectrally majorized.

While the paper contributes to the understanding of optimal biorthogonal filter banks, it is also clear that there are some key unsolved issues. To summarize briefly, consider the prefiltered orthonormal subband coder of Fig. 11, which is a special case of the biorthogonal subband coder. For this case, the optimal choice of filters has been presented in [7] where it was shown that the prefilter $P\left(e^{j \omega}\right)$ and the orthonormal system $\left\{P_{k}\left(e^{j \omega}\right)\right\}$ could be chosen independently. Namely, the prefilter has to be the half-whitening filter for $x(n)$, and $\left\{P_{k}\left(e^{j \omega}\right)\right\}$ the optimal system for the output of the prefilter. For this system the optimal $\phi$ is given by $\phi=\phi_{\eta}$ where $\phi_{\eta}$ is defined in (17).

All evidence in this paper seems to indicate that the most general optimal biorthogonal subband coder can also be represented in this form. This is equivalent to the statement that $\phi \geq \phi_{\eta}$ for any biorthogonal filter bank. Indeed, we could prove this for special classes of psd such as the one demonstrated in Fig. 13(a) (see Theorem 3). However, this has not yet been proved for arbitrary input psd (nor has a counterexample been found yet). For the most general psd, we could only derive bounds on the quantity $\phi$ in terms of the polyphase matrix $\mathbf{E}\left(e^{j \omega}\right)$ (Section $\mathrm{V}$ ). A weaker bound which depends only on the psd $\mathbf{S}_{x x}\left(e^{j \omega}\right)$ but not on $\mathbf{E}\left(e^{j \omega}\right)$ was presented in Section VII, and gave rise to two conclusions (Theorem 3 and Corollary 2).

To summarize the various ways to look at the open problem, consider the polyphase form of the subband coder in Fig. 2. Using the singular value decomposition theorem, we can always redraw this as in Fig. 12 where $\mathbf{U}\left(e^{j \omega}\right)$ and $\mathbf{V}\left(e^{j \omega}\right)$ are paraunitay. In order to prove that the best prefiltered orthonormal system is also the best biorthogonal system, we have to show one of the following.

1) There is an optimal solution such that the output of $\mathbf{U}\left(e^{j \omega}\right)$ is totally decorrelated (Section VI).

2) There is no need for $\mathbf{V}\left(e^{j \omega}\right)$, that is, there is no loss of optimality in setting $\mathbf{V}\left(e^{j \omega}\right)=\mathbf{I}$ and optimizing $\mathbf{U}\left(e^{j \omega}\right)$ and $\lambda_{i}\left(e^{j \omega}\right)$ (Section VI).

3) $\phi \geq \phi_{\eta}$ for any $\mathbf{E}\left(e^{j \omega}\right)$, whenever the psd matrix $\mathbf{S}_{x x}\left(e^{j \omega}\right)$ is diagonal. In view of the decoupling property 
described in Section IV, this is sufficient to show that $\phi \geq \phi_{\eta}$ for any input psd and any biorthogonal filter bank.

4) There exists an optimal solution such that the matrix $\mathbf{E}\left(e^{j \omega}\right) \mathbf{S}_{x x}^{1 / 2}\left(e^{j \omega}\right) \mathbf{E}^{-1}\left(e^{j \omega}\right)$ is Hermitian. (If this is shown, then we apply Corollary 1 in (24), proving $\phi \geq \phi_{\eta}$ and we are done.)

5) There exists an optimal solution such that all inequalities in (23) hold with equality. (If this is shown, then the matrix $\mathbf{E}\left(e^{j \omega}\right) \mathbf{S}_{x x}^{1 / 2}\left(e^{j \omega}\right) \mathbf{E}^{-1}\left(e^{j \omega}\right)$ in (24) can be replaced with a Hermitian one as shown in Section $\mathrm{V}-\mathrm{A}$, and we are done.)

Of course, it is still conceivable that the best prefiltered orthonormal system is not the best biorthogonal system, in which case the open problem is to find the latter. The above special cases and partial solutions appear to be quite insightful, and might even hold the key for a complete solution to the optimal biorthogonal subband coder problem.

\section{APPENDIX I}

\section{DERIVATION OF OBJECTIVE FUNCTION EXPRESSION}

In Fig. 2, the power spectral matrix of the error $\mathbf{e}(n)$ is $\mathbf{S}_{e e}\left(e^{j \omega}\right)=\mathbf{R}\left(e^{j \omega}\right) \mathbf{S}_{q q}\left(e^{j \omega}\right) \mathbf{R}^{\dagger}\left(e^{j \omega}\right)$, where $\mathbf{S}_{q q}\left(e^{j \omega}\right)$ is a constant diagonal matrix with diagonal elements $\sigma_{q_{i}}^{2}$. Thus,

$$
\begin{aligned}
\mathcal{E} & \triangleq E\left[\mathbf{e}^{\dagger}(n) \mathbf{e}(n)\right]=\operatorname{Tr}\left(E\left[\mathbf{e}(n) \mathbf{e}^{\dagger}(n)\right]\right) \\
& =\int_{0}^{2 \pi} \operatorname{Tr} \mathbf{S}_{e e}\left(e^{j \omega}\right) d \omega / 2 \pi \\
& =\int_{0}^{2 \pi} \operatorname{Tr}\left(\mathbf{R}\left(e^{j \omega}\right) \mathbf{S}_{q q}\left(e^{j \omega}\right) \mathbf{R}^{\dagger}\left(e^{j \omega}\right)\right) d \omega / 2 \pi \\
& =\int_{0}^{2 \pi} \operatorname{Tr}\left(\mathbf{R}^{\dagger}\left(e^{j \omega}\right) \mathbf{R}\left(e^{j \omega}\right) \mathbf{S}_{q q}\left(e^{j \omega}\right)\right) d \omega / 2 \pi \\
& =\sum_{i=0}^{M-1} \sigma_{q_{i}}^{2} \int_{0}^{2 \pi}\left(\mathbf{R}^{\dagger}\left(e^{j \omega}\right) \mathbf{R}\left(e^{j \omega}\right)\right)_{i i} d \omega / 2 \pi .
\end{aligned}
$$

Using $\sigma_{q_{i}}^{2}=c 2^{-2 b_{i}} \sigma_{y_{i}}^{2}$ (Section II), we get $\sigma_{q_{i}}^{2}=$ $c 2^{-2 b_{i}} \int_{0}^{2 \pi}\left(\mathbf{E}\left(e^{j \omega}\right) \mathbf{S}_{x x}\left(e^{j \omega}\right) \mathbf{E}^{\dagger}\left(e^{j \omega}\right)\right)_{i i} d \omega / 2 \pi$. Substituting into the preceding equation, this yields

$$
\begin{aligned}
\mathcal{E}= & \sum_{i=0}^{M-1} c 2^{-2 b_{i}} \int_{0}^{2 \pi}\left(\mathbf{E}\left(e^{j \omega}\right) \mathbf{S}_{x x}\left(e^{j \omega}\right) \mathbf{E}^{\dagger}\left(e^{j \omega}\right)\right)_{i i} d \omega / 2 \pi \\
& \times \int_{0}^{2 \pi}\left(\mathbf{R}^{\dagger}\left(e^{j \omega}\right) \mathbf{R}\left(e^{j \omega}\right)\right)_{i i} d \omega / 2 \pi \\
\geq & c M 2^{-2 b} \prod_{i=0}^{M-1}\left(\int_{0}^{2 \pi}\left(\mathbf{E}\left(e^{j \omega}\right) \mathbf{S}_{x x}\left(e^{j \omega}\right) \mathbf{E}^{\dagger}\left(e^{j \omega}\right)\right)_{i i} d \omega / 2 \pi\right. \\
& \left.\times \int_{0}^{2 \pi}\left(\mathbf{R}^{\dagger}\left(e^{j \omega}\right) \mathbf{R}\left(e^{j \omega}\right)\right)_{i i} d \omega / 2 \pi\right)^{1 / M}
\end{aligned}
$$

where we have used the arithmetic-mean/geometric-mean inequality and the definition $b=\sum_{i} b_{i} / M$. This becomes an equality if the terms in the summation are identical for all $i$. This can be accomplished by choosing $b_{i}$ such that

$$
b_{i}=b+0.5 \log _{2} \sigma_{y_{i}}^{2} K_{i}^{2}-0.5 \sum_{i} \log _{2} \sigma_{y_{i}}^{2} K_{i}^{2} / M
$$

where $\sigma_{y_{i}}^{2}=\int_{0}^{2 \pi}\left(\mathbf{E}\left(e^{j \omega}\right) \mathbf{S}_{x x}\left(e^{j \omega}\right) \mathbf{E}^{\dagger}\left(e^{j \omega}\right)\right)_{i i} d \omega / 2 \pi$ and $K_{i}^{2}=\int_{0}^{2 \pi}\left(\mathbf{R}^{\dagger}\left(e^{j \omega}\right) \mathbf{R}\left(e^{j \omega}\right)\right)_{i i} d \omega / 2 \pi$. Equation (41) is called the optimal bit allocation formula [11], [23]. Equation (1) is therefore justified.

\section{APPENDIX II}

\section{ERROR BALANCE PROPERTY}

Let $\mathbf{T}\left(e^{j \omega}\right)$ be a system with WSS vector input $\mathbf{u}(n)$ and output $\mathbf{v}(n)$, having psd matrices $\mathbf{S}_{u u}\left(e^{j \omega}\right)$ and $\mathbf{S}_{v v}\left(e^{j \omega}\right)$. Then $E\left[\mathbf{v}^{\dagger}(n) \mathbf{v}(n)\right]=\operatorname{Tr}\left(E\left[\mathbf{v}(n) \mathbf{v}^{\dagger}(n)\right]\right)=\int_{0}^{2 \pi} \operatorname{Tr}$ $\mathbf{S}_{v v}\left(e^{j \omega}\right) d \omega / 2 \pi$. Similarly $E\left[\mathbf{u}^{\dagger}(n) \mathbf{u}(n)\right]=\int_{0}^{2 \pi} \operatorname{Tr} \mathbf{S}_{u u}\left(e^{j \omega}\right)$ $d \omega / 2 \pi$. This shows $E\left[\mathbf{v}^{\dagger}(n) \mathbf{v}(n)\right]=E\left[\mathbf{u}^{\dagger}(n) \mathbf{u}(n)\right]$ if $\mathbf{T}\left(e^{j \omega}\right)$ is unitary, since $\operatorname{Tr} \mathbf{S}_{v v}\left(e^{j \omega}\right)=\operatorname{Tr}\left(\mathbf{T}\left(e^{j \omega}\right) \mathbf{S}_{u u}\left(e^{j \omega}\right)\right.$ $\left.\mathbf{T}^{\dagger}\left(e^{j \omega}\right)\right)=\operatorname{Tr}\left(\mathbf{T}^{\dagger}\left(e^{j \omega}\right) \mathbf{T}\left(e^{j \omega}\right) \mathbf{S}_{u u}\left(e^{j \omega}\right)\right)=\operatorname{Tr} \mathbf{S}_{u u}\left(e^{j \omega}\right)$.

\section{APPENDIX III}

In [1] the authors state a lower bound $\phi_{\min }$ on $\phi$ (Theorem 2 in [1], with different notations). In our language, this bound is exactly the expression (17), which is the minimum value of $\phi$ achieved by the system of Fig. 8(c). However, the proof of Theorem 2 in [1] appears to have an error [15]. Let us denote the unnumbered equation in [1, p. 1184 (top righthand column)] as "Eq. (*)." The second inequality in that equation is justified by [1, Lemma 1, p. 1183] proved for Hermitian positive definite matrices. But the matrix $\mathbf{T} \mathbf{Q}^{1 / 2} \mathbf{T}^{-1}$ in "Eq. (*)" is not Hermitian in general; it becomes Hermitian only when the first inequality in "Eq. (*)" also holds with equality. Since this would be a side constraint, the claim $\left(\prod_{i} K_{i i} W_{i i}\right)^{1 / m} \geq \bar{\mu}_{g}^{2}$ is not justified, in general [15].

\section{APPENDIX IV \\ EQUALITY ANALYSIS}

The CS-1 inequality in (23) is satisfied with equality if and only if

$$
\left(\mathbf{E}\left(e^{j \omega}\right) \mathbf{S}_{x x}\left(e^{j \omega}\right) \mathbf{E}^{\dagger}\left(e^{j \omega}\right)\right)_{i i}=c_{i}\left(\mathbf{R}^{\dagger}\left(e^{j \omega}\right) \mathbf{R}\left(e^{j \omega}\right)\right)_{i i}
$$

where $c_{i}$ is independent of $\omega$. The CS-2 inequality holds with equality if and only if $\mathbf{S}_{x x}^{1 / 2}\left(e^{j \omega}\right) \mathbf{e}_{i}\left(e^{j \omega}\right)=d_{i}\left(e^{j \omega}\right) \mathbf{r}_{i}\left(e^{j \omega}\right)$. Since this has to hold for all $i$, this means $\mathbf{S}_{x x}^{1 / 2}\left(e^{j \omega}\right) \mathbf{E}^{\dagger}\left(e^{j \omega}\right)=$ $\mathbf{R}\left(e^{j \omega}\right) \mathcal{D}\left(e^{j \omega}\right)$, where $\mathcal{D}\left(e^{j \omega}\right)$ is a diagonal matrix with diagonal elements $d_{i}\left(e^{j \omega}\right)$. Using $\mathbf{R}^{-1}\left(e^{j \omega}\right)=\mathbf{E}\left(e^{j \omega}\right)$, this is equivalent to

$$
\mathbf{E}\left(e^{j \omega}\right) \mathbf{S}_{x x}^{1 / 2}\left(e^{j \omega}\right) \mathbf{E}^{\dagger}\left(e^{j \omega}\right)=\mathcal{D}\left(e^{j \omega}\right)
$$

From $\mathbf{S}_{x x}^{1 / 2}\left(e^{j \omega}\right) \mathbf{E}^{\dagger}\left(e^{j \omega}\right)=\mathbf{R}\left(e^{j \omega}\right) \mathcal{D}\left(e^{j \omega}\right)$, we also obtain

$\mathbf{E}\left(e^{j \omega}\right) \mathbf{S}_{x x}\left(e^{j \omega}\right) \mathbf{E}^{\dagger}\left(e^{j \omega}\right)=\mathcal{D}^{\dagger}\left(e^{j \omega}\right) \mathbf{R}^{\dagger}\left(e^{j \omega}\right) \mathbf{R}\left(e^{j \omega}\right) \mathcal{D}\left(e^{j \omega}\right)$,

which implies, in particular, $\left(\mathbf{E}\left(e^{j \omega}\right) \mathbf{S}_{x x}\left(e^{j \omega}\right) \mathbf{E}^{\dagger}\left(e^{j \omega}\right)\right)_{i i}=$ $\left|d_{i}\left(e^{j \omega}\right)\right|^{2}\left(\mathbf{R}^{\dagger}\left(e^{j \omega}\right) \mathbf{R}\left(e^{j \omega}\right)\right)_{i i}$. Comparing with (42), we conclude that if both $\mathrm{CS}$-inequalities have to hold with equality, we have to have $\left|d_{i}\left(e^{j \omega}\right)\right|^{2}=c_{i}$ independent of $\omega$. Since the left side of (43) is Hermitian positive definite, $d_{i}\left(e^{j \omega}\right)$ are real and positive which shows that $d_{i}\left(e^{j \omega}\right)=\sqrt{c_{i}}>$ 0 . Summarizing, the inequalities CS-1 and CS-2 hold with 


$$
\begin{aligned}
\phi_{\text {new }}= & \left(\prod_{i=0}^{M-1} \int_{0}^{2 \pi} \sqrt{\left(\mathbf{E}_{\text {new }}\left(e^{j \omega}\right) \mathbf{S}_{x x}\left(e^{j \omega}\right) \mathbf{E}_{\text {new }}^{\dagger}\left(e^{j \omega}\right)\right)_{i i}\left(\mathbf{R}_{\text {new }}^{\dagger}\left(e^{j \omega}\right) \mathbf{R}_{\text {new }}\left(e^{j \omega}\right)\right)_{i i}} d \omega / 2 \pi\right)^{2} \\
= & \left(\prod_{i=0}^{M-1} \int_{0}^{2 \pi} \sqrt{\left(\mathbf{E}\left(e^{j \omega}\right) \mathbf{S}_{x x}\left(e^{j \omega}\right) \mathbf{E}^{\dagger}\left(e^{j \omega}\right)\right)_{i i}\left(\mathbf{R}^{\dagger}\left(e^{j \omega}\right) \mathbf{R}\left(e^{j \omega}\right)\right)_{i i}} d \omega / 2 \pi\right)^{2} \\
\leq & \prod_{i=0}^{M-1} \int_{0}^{2 \pi}\left(\mathbf{E}\left(e^{j \omega}\right) \mathbf{S}_{x x}\left(e^{j \omega}\right) \mathbf{E}^{\dagger}\left(e^{j \omega}\right)\right)_{i i} d \omega / 2 \pi \\
& \times \int_{0}^{2 \pi}\left(\mathbf{R}^{\dagger}\left(e^{j \omega}\right) \mathbf{R}\left(e^{j \omega}\right)\right)_{i i} d \omega / 2 \pi=\phi
\end{aligned}
$$

equality if and only if $\mathbf{E}\left(e^{j \omega}\right) \mathbf{S}_{x x}^{1 / 2}\left(e^{j \omega}\right) \mathbf{E}^{\dagger}\left(e^{j \omega}\right)=\mathcal{D}$ for some diagonal matrix $\mathcal{D}$ with constant diagonal elements $d_{i}>0$.

We now show that this condition automatically implies that the third inequality in (23) becomes an equality. The equality condition (43) can be rewritten as $\mathbf{E}\left(e^{j \omega}\right) \mathbf{S}_{x x}^{1 / 2}\left(e^{j \omega}\right)=$ $\mathcal{D} \mathbf{R}^{\dagger}\left(e^{j \omega}\right)$ which implies $\mathbf{E}\left(e^{j \omega}\right) \mathbf{S}_{x x}^{1 / 2}\left(e^{j \omega}\right) \mathbf{R}\left(e^{j \omega}\right)=\mathcal{D} \mathbf{R}^{\dagger}$ $\left(e^{j \omega}\right) \mathbf{R}\left(e^{j \omega}\right)$. Thus the quantity

$$
\begin{aligned}
& \mathbf{e}_{i}^{\dagger}\left(e^{j \omega}\right) \mathbf{S}_{x x}^{1 / 2}\left(e^{j \omega}\right) \mathbf{r}_{i}\left(e^{j \omega}\right) \\
& \quad=\left(\mathbf{E}\left(e^{j \omega}\right) \mathbf{S}_{x x}^{1 / 2}\left(e^{j \omega}\right) \mathbf{R}\left(e^{j \omega}\right)\right)_{i i}=d_{i}\left\|\mathbf{r}_{i}\right\|^{2}>0
\end{aligned}
$$

showing that the third inequality in (23) automatically holds with equality.

At the beginning of Section V-A we mentioned that there is a loss of generality if we force the two CS-inequalities to be equalities. However, the CS-1 inequality can be forced to be an equality without loss of generality. The reason is as follows. Given any $\mathbf{E}\left(e^{j \omega}\right)$ and $\mathbf{R}\left(e^{j \omega}\right)$, define the new polyphase matrices $\mathbf{E}_{\text {new }}\left(e^{j \omega}\right)=\mathcal{F}\left(e^{j \omega}\right) \mathbf{E}\left(e^{j \omega}\right)$ and $\mathbf{R}_{\text {new }}\left(e^{j \omega}\right)=$ $\mathbf{R}\left(e^{j \omega}\right) \mathcal{F}^{-1}\left(e^{j \omega}\right)$ where $\mathcal{F}\left(e^{j \omega}\right)$ is a diagonal matrix. By appropriate choice of the diagonal elements $f_{i}\left(e^{j \omega}\right)$, namely such that

$$
\left|f_{i}\left(e^{j \omega}\right)\right|^{4}=\frac{\left(\mathbf{R}^{\dagger}\left(e^{j \omega}\right) \mathbf{R}\left(e^{j \omega}\right)\right)_{i i}}{\left(\mathbf{E}\left(e^{j \omega}\right) \mathbf{S}_{x x}\left(e^{j \omega}\right) \mathbf{E}^{\dagger}\left(e^{j \omega}\right)\right)_{i i}}
$$

we ensure that the new system satisfies (42). We then have (45) as shown at the top of the page. That is, if $\mathbf{E}\left(e^{j \omega}\right)$ does not satisfy the first equality condition (42), we can replace it with $\mathbf{E}_{\text {new }}\left(e^{j \omega}\right)$ which satisfies that condition and has smaller $\phi$. So, without loss of generality, we can assume that the optimal biorthogonal system satisfies (42), and that $\phi$ has the form (29). One corollary of this observation is this: suppose the optimal system does satisfy (43). Then the diagonal matrix $\mathcal{D}\left(e^{j \omega}\right)$ can be assumed to be a constant $\mathcal{D}$.

\section{ACKNOWLEDGMENT}

The authors are grateful to Prof. T. Chen, Dr. C. Creusere, and Prof. N. Erdol, who read the manuscript very carefully and provided useful comments. The authors would also like to thank Prof. C. T. Mullis and Dr. K. Aas for discussions pertaining to [1]. The authors wish to thank Prof. J. Choma, Jr. for his invitation to write this article.

\section{REFERENCES}

[1] K. C. Aas and C. T. Mullis, "Minimum mean-squared error transform coding and subband coding," IEEE Trans. Inform. Theory, pp. 1179-1192, July 1996.

[2] S. O. Aase and T. Ramstad, "On the optimality of nonunitary filter banks in subband coders," IEEE Trans. Image Processing, pp. 1585-1591, Dec. 1995.

[3] A. N. Akansu and Y. Liu, "On signal decomposition techniques," Opt. Eng.., vol. 30, July 1991.

[4] M. Antonini, M. Barland, P. Mathieu, and I. Daubechies, "Image coding using wavelet transform," IEEE Trans. Image Processing, pp. 205-220, Apr. 1992.

[5] P. Delsarte, B. Macq, and D. Slock, "Signal-adapted multiresolution transform for image coding," IEEE Trans. Inform. Theory, vol. 38, pp. 897-904, Mar. 1992

[6] R. L. de Queiroz and H. S. Malvar, "On the asymptotic performance of hierarchical transforms," IEEE Trans. Signal Processing, vol. 40, pp. 2620-2622, Oct. 1992.

[7] I. Djokovic and P. P. Vaidyanathan, "On optimal analysis/synthesis filters for coding gain optimization," IEEE Trans. Signal Processing, pp. 1276-1279, May 1996.

[8] R. A. Haddad and K. Park, "Modeling, analysis, and optimum design of quantized $M$-band filter banks," IEEE Trans. Signal Processing, pp. 2540-2549, Nov. 1995.

[9] R. A. Horn and C. R. Johnson, Matrix Analysis. Cambridge, UK: University Press, 1985.

[10] Y. Huang and P. M. Schultheiss, "Block quantization of correlated Gaussian random variables," IEEE Trans. Commun. Syst., pp. 289-296, Sept. 1963.

[11] N. S. Jayant and P. Noll, Digital Coding of Waveforms. Englewood Cliffs, NJ: Prentice-Hall, 1984.

[12] A. Kirac and P. P. Vaidyanathan, "Theory and design of optimum FIR compaction filters," IEEE Trans. Signal Processing (Special Issue on Theory and Applications of Filter Banks and Wavelet Transforms), 1998.

[13] H. S. Malvar, Signal Processing with Lapped Transforms. Norwood, MA: Artech House, 1992.

[14] P. Moulin, "A new look at signal-adapted QMF bank design," in Proc. Int. Conf. ASSP, Detroit, May 1995, pp. 1312-1315.

[15] C. T. Mullis and K. Aas, private communication.

[16] R. P. Rao and W. A. Pearlman, "On entropy of pyramid structures," IEEE Trans. Inform. Theory, vol. 37, pp. 407-413, Mar. 1991.

[17] V. Sathe and P. P. Vaidyanathan, "Effects of multirate systems on the statistical properties of random signals," IEEE Trans. Signal Processing, pp. 131-146, Jan. 1993.

[18] A. Segall, "Bit allocation and encoding for vector sources," IEEE Trans. Inform. Theory, pp. 162-169, Mar. 1976.

[19] M. K. Tsatsanis and G. B. Giannakis, "Principal component filter banks for optimal multiresolution analysis," IEEE Trans. Signal Processing, vol. 43, pp. 1766-1777, Aug. 1995.

[20] J. Tuqan and P. P. Vaidyanathan, "Optimum low cost two channel IIR orthonormal filter bank," in Proc. IEEE Int. Conf. Acoust., Speech, Signal Processing, Munich, Germany, Apr. 1997, pp. 2425-2428. 
[21] M. Unser, "On the optimality of ideal filters for pyramid and wavelet signal approximation," IEEE Trans. Signal Processing, vol. 41, pp. 3591-3596, Dec. 1993.

[22] _ "An extension of the KLT for wavelets and perfect reconstruction filter banks," in Proc. SPIE no. 2034, Wavelet Appl. Signal Image Processing, San Diego, CA, 1993, pp. 45-56.

[23] P. P. Vaidyanathan, Multirate Systems and Filter Banks. Englewood Cliffs, NJ: Prentice-Hall, 1993.

[24] "Theory of optimal orthonormal subband coders," IEEE Trans. Signal Processing, submitted for publication.

[25] "Theory of optimal orthonormal filter banks," in Proc. IEEE Int. Conf. Acoust. Speech, Signal Processing, Atlanta, GA, May 1996.

[26] M. Vetterli and Kovačević, J. Wavelets and Subband Coding. Englewood Cliffs, NJ: Prentice-Hall, 1995.

[27] N. Wiener and P. Masani, "The prediction theory of multivariate stochastic processes-Part I: The regularity condition," Acta. Math., vol. 98, pp. 111-149, 1957.

[28] B. Xuan and R. H. Bamberger, "Complete FIR principal component filter banks," in Proc. ISCAS, Atlanta, May 1996, pp. 417-420.
P. P. Vaidyanathan (S'80-M'83-SM'88-F'91), for a photograph and biography, see this issue, p. 931.

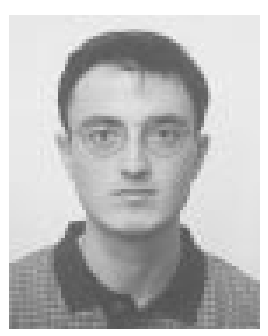

Ahmet Kirac (S'98) was born in Afsin, K. Maras, Turkey, on October 10, 1971. He received the B.S. degree in electrical engineering from Bilkent University, Ankara, Turkey, in 1993 and the M.S degree in electrical engineering from the California Institute of Technology in 1994. He is currently pursuing the Ph.D. degree at the California Institute of Technology, Pasadena, CA. His research interests include digital signal processing, especially multirate systems and filter banks, wavelet transforms, and optimal quantization. 